\title{
Zinc Metabolic Defect of Aging Alveolar Progenitors in Progressive Pulmonary Fibrosis
}

Jiurong Liang1,*, Guanling Huang1, Xue Liu1, Forough Taghavifar1, Ningshan Liu1, Yizhou Wang2, Nan Deng2, Changfu Yao1, Ankita Burman1, Ting Xie1, Simon Rowan1, S. Samuel Weigt3, John Belperio3, Barry Stripp1, William C. Parks1,4, Dianhua Jiang 1,4,5, and Paul W. Noble1,5,6,

1Department of Medicine and Women's Guild Lung Institute, Cedars-Sinai Medical Center, Los Angeles, CA, USA

2Genomics Core, Cedars-Sinai Medical Center, Los Angeles, CA, USA

103 Department of Medicine, University of California at Los Angees (UCLA), Los Angeles, CA, USA.

4Department of Biomedical Sciences, Cedars-Sinai Medical Center, Los Angeles, CA, USA.

5Senior author

*Correspondence: carol.liang@cshs.org (J.L.), paul.noble@cshs.org (P.W.N.)

In Brief: Liang et al. performed single cell RNA-seq (scRNA-seq) of epithelial cells in IPF and in mice and discovered a zinc metabolic defect of alveolar progenitor cells (AEC2) in IPF and injured old mice characterized by down-regulation of a specific zinc transporter ZIP8. Manipulation of ZIP8 and zinc in 3D organoid culture of AEC2 in vitro and targeted deletion of Zip8 in AEC2s in vivo demonsrated a role of ZIP8 in promoting $A E C 2$ progenitor function.

\section{Highlights:}

- ScRNA-seq revealed dysregulation of zinc metabolism in AEC2s and decreased stem cell signaling in IPF

- Reduced SLC39A8 and related gene expression of IPF AEC2s and aged mouse AEC2s

- ZIP8-dependent zinc metabolism is required for AEC2 renewal

- Targeted deletion of S/c39a8 impaired AEC2 renewal and promoted lung fibrosis

Character Count: 38,158 characters (5,947 words) (excluding methods 11,783 , figure legends 8,273 , and references 14,420). 


\section{SUMMARY}

Idiopathic pulmonary fibrosis (IPF) is a fatal form of interstitial lung disease and aging has been identified as a risk factor to the disease. Alveolar type II cells (AEC2s) function as progenitor cells in the lung. Growing evidences indicate that IPF results from repeating AEC2 injury and inadequate epithelial repair. We previously reported that there was a significant loss of alveolar progenitors in the lungs of patients with IPF. In our current study, we performed single cell RNA-seq of epithelial cells from lungs of patients with IPF and healthy donors as well as epithelial cells from old and young mouse lungs with bleomycin injury. We identified a defect of zinc metabolism of AEC2s from IPF lungs and bleomycininjured old mouse lungs. We further discovered that a specific zinc transporter ZIP8 was down regulated in IPF AEC2s and AEC2s from aged mice. Loss of ZIP8 expression is associated with impaired AEC2 renewal through sirtuin signaling in aging and IPF. Targeted deletion of Zip8 in murine AEC2 compartment led to reduced AEC2 renewal capacity, impaired AEC2 recovery, and worsened lung fibrosis after bleomycin injury. In summary, we have identified novel metabolic defects of AEC2s during aging and in IPF which contribute to the pathogenesis of lung fibrosis. Therapeutic strategies to restore critical components of these metabolic programs could improve AEC2 progenitor activity and mitigate ongoing fibrogenesis.

Key Words: IPF, aging, zinc metabolism, progenitor, fibrosis, 


\section{INTRDUCTION}

Despite extensive efforts, the mechanisms controlling progressive tissue fibrosis remain poorly understood, resulting in the unfortunate lack of effective therapies for patients with progressive tissue fibrosis. Idiopathic pulmonary fibrosis (IPF) is a fatal form of interstitial lung disease. The hallmark of pathogenesis of IPF is repeated epithelial cell injury and inadequate alveolar epithelial repair that leads to excessive fibroblast activation and lung fibrosis (Jiang et al., 2020; Noble et al., 2012).

Type 2 alveolar epithelial cells (AEC2s) function as progenitor cells that maintain epithelium homeostasis and repair damaged epithelium after injury (Barkauskas et al., 2013; Desai et al., 2014; Hogan et al., 2014). We have showed previously that AEC2s were reduced in the lungs of patients with IPF. The remaining AEC2s in the IPF lung have impaired progenitor function and failed to renew themselves adequaly (Liang et al., 2016). However, the molecular mechanisms that control AEC2 stem cell renewal and unremitting lung fibrosis remain poorly understood.

Aging is one of the critical risk factors for IPF. The incidence, prevalence, and mortality of IPF all increase with aging (Raghu et al., 2016; Selman and Pardo, 2014) (Rojas et al., 2015; Thannickal, 2013). Aging has also been linked to lung fibrosis in animal models (Bueno et al., 2018). Phenotypes of cellular aging including epithelial cell apoptosis (Korfei et al., 2008; Lawson et al., 2011), autophagy (Larson-Casey et al., 2016; Sosulski et al., 2015), senescence (Minagawa et al., 2011), telomere shortening (Alder et al., 2008; Naikawadi et al., 2016), mitochondria dysfunction (Bueno et al., 2015; Ryu et al., 2017), and oxidative stress (Anathy et al., 2018) are observed in IPF lungs. Although the concept that IPF is a disease of aging has been well accepted (Martinez et al., 2017; Selman et al., 2016; Thannickal, 2013), there are limited studies focusing on the agerelated mechanisms that contributes to the development and progression of IPF. In this study, we dissected the detrimental role of aging in AEC2 progenitor renewal in IPF.

Multiple metabolic dysfunctions undergo with aging (Bueno et al., 2018; ShyhChang and Ng, 2017; Tevy et al., 2013; Yaku et al., 2018). Recent studies have linked metabolic changes with stem cell fate (Lee and Hong, 2020; Shyh-Chang and Ng, 2017; Zhang et al., 2018). Zinc is an essential element and involvels in maintaining multiple functions of human body (Bogden, 2004). In the lung, reports showed that zinc deficiency exacerbated ventilation-induced lung injury (Boudreault et al., 2017; Chen et al., 2012b). Patients with acute respiratory distress syndrome (ARDS) showed significant lower plasma zinc levels relative to control individuals (Boudreault et al., 2017; Cander et al., 2011). Zinc deficiency increased lung and spleen damage (Knoell et al., 2009) in animal sepsis models. These evidence suggests the importance of zinc metabolism in lung biology. Zinc homeostasis is controlled by two zinc transporter families, $\mathrm{Zn}$ transporters $(\mathrm{ZnT})$ and Zrt-, Irt-related proteins (ZIP) in maintaining zinc mobilization and 
compartmentalization across biological membranes (Kambe et al., 2015). Studies showed that zinc and zinc transporters played an important role in maintenance homeostasis and progenitor function of intestinal epithelium (Ohashi et al., 2019). Zinc is required for sirtuin signaling which is a major cell energy metabolism pathway (Imai and Guarente, 2014; Yaku et al., 2018). Studies showed that sirtuins play a role in stem cells maintenance and differentiation (Nogueiras et al., 2012; O'Callaghan and Vassilopoulos, 100 2017). The role of zinc metabolism in lung stem cell renewal and epithelial repair is largely unknown.

In the current study, using unbiased single cell RNA-sequencing (scRNA-seq), we have found dysregulated metabolic pathways of AEC2s from patients with IPF. We discovered a significant decrease of a zinc transporter SLC39A8 (ZIP8) in IPF AEC2s. We further demonstrated that loss of ZIP8 in IPF AEC2s resulted in impaired progenitor function of the cells via SIRT1. Exogenous zinc treatment increased ZIP8 and SIRT1 expression and promoted renewal capacity of AEC2s. With mouse model of aging, we have found that AEC2s from bleomycin injured aged mouse lungs shared the similar genomic changes and cellular phenotypes with IPF AEC2s. AEC2s from the lungs of aged 110 mice showed reduced Zip8 expression and decreased renewal capacity relative to that of $A E C 2 s$ from the lungs of young mice. Exogenous zinc was able to promote renewal and differentiation of murine AEC2s while the response to zinc treatment blunted with aging. With genomic modified mice, we further demonstrated that targeted deletion of ZIP8 in AEC2s compartment impaired AEC2s recovery and worsened lung fibrosis after

115 bleomycin injury. To our knowledge this is the first evidence to show that zinc transporter ZIP8 and zinc metabolism play a role in regulating AEC2 progenitor renewal and lung fibrosis. 


\section{RESULTS}

\section{ScRNA-seq revealed the loss of AEC2s in IPF lungs}

In the previous study, we found a significant reduction of AEC2 population in patients with IPF (Liang et al., 2016). The remaining AEC2s in the diseased lung had impaired renewal capacity compared to that of the AEC2s from healthy donor lung (Liang et al., 2016). To further investigate the genomic changes of AEC2s in IPF, we performed scRNA-seq of flow cytometry-enriched epithelial (Lin-EpCAM+) cells from lung tissues of IPF patients and healthy donors.

We processed single cell homogenates of lungs from six individuals in each group and that contained 11,381 cells from healthy individuals and 14,687 cells from IPF patients, after removing cells with low quality of RNA. The Uniform Manifold Approximation and Projection (UMAP) plots were generated with a Seurat package and the cells from all samples were overlapped very well (Figure S1A, S1B). The major lung epithelial cell types, AEC2, AEC1, basal cell, Club cell, ciliated cell, pulmonary neuroendocrine cell (PNEC), and proliferative (cycling) cells were readily identified with classical cell markers (Figure S1C).

When we compared the cells from IPF lungs to the cells form healthy lungs, we observed much fewer cells in AEC2 cluster of IPF lungs compared to that of the healthy lungs (Figure 1A, 1B). Increased basal-like cells and ciliated cells were observed with the cells from IPF lungs (Figure 1A, 1B), consistent with recent reports (Adams et al., 2020; Habermann et al., 2020; Xu et al., 2016) . The investigation into the biological significance of the accumulation of basal and ciliated cells in IPF pathogenesis is under way in the laboratory. In this study, we focus on the investigation of molecular mechanism which causes dysfunction of AEC2 progenitors in IPF. Expression of AEC2 marker genes including SFTPC, SFTPA2, SFTPB, and ABCA3 were all down regulated in IPF AEC2s relative to healthy AEC2s (Figure 1C). We confirmed decreased SFTPC expression of flow sorted AEC2s from IPF lungs relative to AEC2s from healthy donor lungs using real time qPCR (Figure 1D).

\section{Dysregulation of metabolism and stem cell signaling in IPF AEC2s}

Next, we performed the pathway analysis to compare gene expression of AEC2s from 150 IPF lungs and healthy lungs and found that several metabolic pathways were dysregulated. Based on the findings of our previous study that the AEC2 progenitor function faltered in IPF lungs, we would expect to see the dysregulated stem cell/progenitor cell related signaling in IPF AEC2s. Indeed, we found that the sirtuin signaling pathway was on the top of the list of down regulated pathways of IPF AEC2s 
155 (Figure 1E). IPF AEC2s showed lower SIRT score (Figure 1F). The sirtuin signaling is considered as metabolic master switches that control several aspects of metabolism, including glycolysis and fatty acid, lipid, and redox processes (Hershberger et al., 2017). Pathways of NRF2-mediated oxidative stress response and glutathione redox reactions I were down regulated in IPF AEC2s. Down regulation of PTEN signaling in IPF AEC2s (Figure 1E) was consitent with the previous reports (Tian et al., 2019).

The function of the sirtuin signaling has been linked to stem cell renewal and differentiation (Nogueiras et al., 2012; O'Callaghan and Vassilopoulos, 2017). To test the role of sirtuin signaling in regulating AEC2 progenitor renewal, we applied Sirtuin activator, SRT1720, and sirtuin inhibitor, splitomicin, to 3D organoid culture of AEC2s.

165 Our results showed that SRT1720 promoted and splitomicin inhibited colony formation of both human (Figure 1G) and mouse AEC2s (Figure S2A,2B), suggesting sirtuin signaling is important for AEC2 progenitor renewal. Sirt1 is the most important member of sirtuin family (Leibiger and Berggren, 2006; Price et al., 2012). We found decreased expression of SIRT1 in IPF AEC2s both fresh isolated from lung tissues (Figure $1 \mathrm{H}$ ) and derived from

$3 D$ cultured organoids (Figure 1I) relative to that of the AEC2s from healthy lungs using qPCR. These data suggest that dysregulated metabolic programs of IPF AEC2s leads to progenitor function failure of the cells, and lower SIRT activity in IPF AEC2s may contribute to their lower renewal potential.

\section{Reduced expression of SLC39A8 and zinc metabolism related genes of IPF AEC2s}

Sirtuin signaling is zinc dependent cell energy metabolism pathway, and SIRT enzymatic activities require an adequate level of intracellular zinc (Nogueiras et al., 2012). Next we looked at the expression of zinc metabolism related genes of AEC2s from IPF patient and healthy donor lungs in our single cell RNA-sq data set. We found several zinc metabolism

180 related genes including MT1E, GCLM, and GSR were all down regulated in IPF AEC2s relative to that of AEC2s from healthy lungs (Figure $2 A$ ). These data suggested that there was a defect of zinc metabolism with AEC2s from IPF lungs and down regulation of sirtuin signaling of IPF AEC2s might result from zinc metabolic dysfunction of the cells.

Zinc homeostasis is controlled by cell membrane bound zinc transporters (Kambe 185 et al., 2015). Most zinc transporters are tissue specific and cell type specific (Kambe et al., 2015). SLC39 gene family encodes zinc influx transporters, ZIP proteins. There are total 14 members in the ZIP protein family. We looked at the gene expression of ZIP proteins in lung tissue with online deposited datasets (Fagerberg et al., 2014) and found SLC39A8 (encoding ZIP8) was highly expressed in the lung (Figure S3A). Lung tissue

190 expresses SLC39A8 the highest compared to that of other organs or tissues (Figure S 3B). 
Next we tried to determine cell types that express SLC39A8 in the lung by analyzing our human lung scRNA-seq data. Within lung epithelial cells, SLC39A8 mainly expressed in alveolar type I cells (AEC1s) and AEC2s (Figure 2B). We then compared SLC39A8 expression level of AEC2s between healthy and IPF and found AEC2s from IPF lungs expressed much lower SLC39A8 than that of AEC2s from healthy lungs (Figure 2C). We confirmed decreased SLC39A8 expression of IPF AEC2s both directly isolated from lung tissues (Figure 2D) and derived from 3D cultured organoids (Figure 2E) using qPCR. We further showed that cell surface expression of ZIP8 protein of IPF AEC2s was 200 decreased compared to that of healthy AEC2s using flow cytometry (Figure 2G, $2 \mathrm{H}$ ). We analyzed LGRC deposited data and found SLC39A8 expression was significantly lower in lung tissues of patients with interstitial lung diseases (ILD) (Figure 2I), which is consistent with our findings of decreased SLC39A8 expression in IPF AEC2s.

SLC39A8 expression was also decreased in AEC1s from IPF lungs relative to that of AEC1s from healthy lungs (Figure S3C). The significance of ZIP8 in AEC1s is under investigation and not the focus of this study. The expression of SLC39A1 and SLC39A7 are relatively high in addition to $S L C 39 A 8$ in the lung. We found that the expression of SLC39A1 was slightly lower in IPF AEC2s (Figure S3D) and SLC39A7 expression was similar between AEC2s from healthy and IPF lungs (Figure S3D). ZNT family genes were

210 expressed in human AEC2s at a lower level than that of ZIP family genes (Figure S3E). The expression of SLC30A1, SLC30A6, SLC30A7 are slightly lower in IPF AEC2s compared to healthy AEC2s (Figure S3E). SLC30A5 and SLC30A9 expression showed no significant difference between IPF and healthy AEC2s (Figure S3E). Other ZNT family members were not expressed.

Furthermore, IPF AEC2s showed lower intracellular zinc levels compared to that of AEC2s from healthy lungs (Figure 2J). Lower levels of intracellular zinc with IPF AEC2s possibly result from decreased ZIP8 expression of the cells. These data identified a dysregulated zinc matabolism in IPF AEC2s characterized by the loss of SLC39A8/ZIP8 expression and lower intracelluar zinc levels.

\section{ZIP8-dependent zinc metabolism is required for AEC2 renewal}

Zinc is an essential element for cell metabolism and involves in various cell functions. It has been reported that zinc metabolism has a role in maintaining epithelial progenitor function (Ohashi et al., 2019; Ohashi et al., 2016; Yang et al., 2020). We hypothesized that ZIP8, as the zinc influx transporter of AEC2s, would play an important role in regulating AEC2 progenitor function. To test this hypothesis, we flow sorted ZIP8pos and ZIP8neg AEC2s from both healthy and IPF lungs, applied the cells to 3D organoid culture, and determined the renewal capacity of AEC2s measured by colony forming efficiency 
(CFE) (Figure 3A, 3B). ZIP8neg AEC2s showed significantly lower renewal potential compared to that of ZIP8pos AEC2s from the same lung (Figure 3B). Both cells from healthy lungs and IPF lungs showed the same pattern even though ZIP8pos AEC2s from IPF lungs still had lower CFE than the ZIP8pos AEC2s from healthy lungs did (Figure 3B), indicating there might be other factors contribute to impaired renewal of IPF AEC2s. AEC2s derived from colonies of ZIP8neg AEC2s showed reduced SIRT1 expression 235 relative to that of ZIP8pos AEC2s (Figure $3 C$ ) suggesting that zinc homeostasis directly affected sirtuin signaling of AEC2s. ZIP8neg AEC2s also had lower PDPN expression indicating that ZIP8 deficiency might also affect AEC2 to AEC1 differentiation (Figure 3D).

Next we tested weather exogenous zinc affects AEC2 progenitor renewal. As proof-of-principle, we applied $100 \mu \mathrm{M}$ zinc sulfate $\left(\mathrm{ZnSO}_{4}\right)$ into $3 \mathrm{D}$ organoid culture of AEC2s and zinc treatment was able to improve the colony forming capacities of AEC2s from both healthy and IPF lungs (Figure 3E). However, zinc treatment showed no effect on CFE of ZIP8neg AEC2s (Figure 3F), indicating ZIP8 might act as a sole functional zinc transport of AEC2s.

To verify the specificity of exogenous zinc treatment on promoting AEC2 progenitor renewal, we applied the zinc chelator, TPEN to the 3D organoid culture. TPEN was able to abolish the effect of zinc treatment in promoting AEC2 renewal (Figure 3G).

Zinc treatment elevated SLC39A8 expression of AEC2 from both healthy and IPF lungs (Figure $3 \mathrm{H}$ ). AEC2s derived from $3 \mathrm{D}$ organoids with zinc treatment also showed increased SIRT1 (Figure 3I) and SFTPC (Figure 3J) expression. AEC2s from healthy

250 lungs showed much better response to zinc treatment than the AEC2s from IPF lungs did (Figure $3 \mathrm{H}-3 \mathrm{~J}$ ).

We have showed previously that a loss of cells surface hyaluronan (HA) of AEC2s in IPF lungs contributed to the impaired renewal capacity of the cells (Liang et al., 2016). Since loss of HA and loss of ZIP8 both happened with IPF AEC2s, we would expect a correlation between ZIP8 expression and cell surface HA levels of AEC2s. We compared HA expression of ZIP8+ and ZIP8- AEC2s from both healthy and IPF lungs using flow cytometry and ZIP8+ AEC2s showed higher HA levels relative to the ZIP8- AEC2s from the same lung (Figure S4A). Zinc treatment was able to elevate cell surface HA of AEC2s (Figure S4B).

\section{Reduced renewal capacity of AEC2s from aged mice}

Aging is one of the important risk factors to IPF pathogenesis. Next we investigated how aging affect AEC2 progenitor functions. We accessed epithelial cells from the lungs of 2 -3 mouths old (young) and $18-20$ months old (aged) mice by flow cytometry using cell surface marker gating strategy established in the lab (Liang et al., 2016). Epithelial cells are CD31-CD34-CD45-EpCAM+ (R1), whereas AEC2s are CD31-CD34-CD45-EpCAM+ 
AEC2s (R2) (Figure 4A). The numbers of total cells recovered from each lung were similar between young and old mice (Figure 4B). However, we observed reduced percentage and total number of epithelial cells in old mouse lungs compared to that of young mouse lungs (Figure 4C, 4D). We then looked AEC2s within epithelial population and found the percentage of AEC2s was much lower with the cells from old mouse lungs than the cells from young mouse lungs (Figure 4E), that resulted in extremely low number of AEC2s in the old mouse lungs (Figure 4F).

We further analyzed ZIP8 expression of AEC2s and observed a big reduction of percentage (Figure 4A, 4G) and total number (Figure 4H) of ZIP8+ AEC2s (R3 in Figure $4 A$ ) in old mouse lungs relative to that in young mouse lungs. These data suggested that the loss of ZIP8+ AEC2 progenitor cells might be one of the characteristics of lung aging.

Next we compared the regenerative capacity of AEC2s from young and old mouse lungs with $3 \mathrm{D}$ organoid culture. AEC2s from old mouse lungs gave rise to fewer colonies than the AEC2s from young mouse lungs (Figure 4I). AEC2s derived from colonies of old mouse AEC2s showed lower expression of Sftpc (Figure 4J) and Pdpn (Figure 4K) relative to the cells derived from colonies of young $A E C 2 s$, indicating impaired renewal and differentiation of AEC2 progenitors with aging.

\section{Impaired ZIP8-dependent AEC2 renewal in aging and post injury.}

285 We have performed single cell RNA-seq of flow enriched lung epithelial cells from both young and old mice at uninjured stage (day 0 ) as well as day 4 and day 14 after bleomycin injury, which is reported in a separate paper (bioRxiv doi will be updated here). To further investigate the role of aging in regulating ZIP8 expression and progenitor function of AEC2s, we analyzed gene expression of mouse AEC2s from the dataset of mouse lung 290 single cell RNA-seq.

We first performed pathway analysis to compare gene expression program of AEC2s from day 4 bleomycin injured young and old mouse lungs. Similar to what we have observed with IPF AEC2s, we found that the sirtuin signaling pathway was on the top of down regulated pathways with the lowest z-score of $A E C 2 s$ from old mice relative to AEC2s from young mice (Figure 5A). This finding indicated that injured AEC2s in the injured old mouse lungs might share the similar genomic changes with IPF AEC2s.

Similar to what we observed with human lung epithelial cells, S/c39a8 mainly expressed in mouse alveolar epithelial cells (Figure S3F). S/c39a8 expression was down regulated in $A E C 2 s$ from old mice relative to that of $A E C 2 s$ from young mice using q-PCR (Figure 5B). 
Next, we analyzed S/c39a8 expression of AEC2s in the bleomycin injured lungs from young and old mice. At day 4, the timepoint with a maximum of AEC2 injury after bleomycin treatment, Slc39a8 expression was decreased in AEC2s from both young and old mice (Figure 5C). However, the AEC2s from old mouse lungs had more severe loss 305 of S/c39a8 expression relative to that of young AEC2s (Figure 5C). It is interesting that at day 14, the epithelium recovery stage after bleomycin injury, the Slc39a8 expression of ACE2s from young mice was partially recovered, but its expression in AEC2s of old mice remained low (Figure 5C).

Consistent with the pattern of S/c39a8 expression of AEC2s after bleomycin injury,

310 AEC2 marker genes including Sftpc and Abca3 were highly expressed in both young and old AEC2s from day 0 intact lungs (Figure 5D) and these genes were down regulated in both young and old AEC2s at day 4 after bleomycin injury (Figure 5D). It is most interesting that at day 14, the expression of AEC2 marker genes of AEC2s from young mice was recovered, but their expression of AEC2s from old mouse lungs was lingered 315 behind (Figure 5D). The number of AEC2s recovered from day 14 old mice was significantly low than the number of AEC2s from day 14 young mice (Figure 5E). These data suggest that severe loss ZIP8 of AEC2s in old mouse lung significantly hampered AEC2 recovery of aged mice after lung injury.

\section{Blunted response to zinc treatment of AEC2s from old mouse lungs}

Next, we investigated whether exogenous zinc would affect the progenitor function of murine AEC2s. AEC2s with zinc treatment gave rise to an increased number of colonies in $3 D$ organoid culture relative to AEC2s cultured in control medium (Figure 6A). The increased colony formation of AEC2s with zinc treatment could be explained by elevated ZIP8 expression of the cells (Figure 6B,6C).

We anticipated that aging would have impact on the responses of murine AEC2s to exogenous zinc treatment. To test this hypothesis, we isolated AEC2s from multiple age groups of mice including 2.5 months old (young) mice, 18 months old (aged) mice, and 12 months and 14 months old in between (Figure 6D - 6G). We applied the flow sorted AEC2s to 3D organoid culture with zinc treatment. Zinc treatment increased the CFEs of AEC2s from all age groups (Figure 6D). However, ACE2s from 2.5 months old young mouse responded to zinc treatment the best and the responses diminished when the mice got older (Figure 6D). Slc39a8 expression increased in the same pattern as CFE increased with zinc treatment (Figure 6E). AEC2s derived from the organoids with zinc

335 treatment showed increased Sftpc (Figure 6F) and Pdpn (Figure 6G) expression indicating improved progenitor renewal and differentiation of the cells. The response to 
zinc treatment was most significant with AEC2s from 2.5 months old (young) mice and the response blunted when the age of the mice increased (Figure 6D -6G).

\section{Targeted deletion of Slc39a8 in AEC2s decreased AEC2 renewal}

To further illustrate the role of ZIP8 in AEC2 renewal and lung fibrosis in vivo, we generated a mouse line with targeted deletion of Slc39a8 in the AEC2 compartment, SFTPC-CreER;Rosa26-tdTomato;Zip8floxflox (Zip8 $\triangle \mathrm{AEC} 2$ ), by cross breeding SFTPCCreER;Rosa26-tdTomato mice with Slc39a8 floxed mice (acquired from MMRRC

345 Repository). Rosa26-tdTomato is used to facility cell sorting. Upon tamoxifen treatment, Slc39a8 expression in Sftpc-expressing cells is deleted. We confirmed the genotype of the of Zip8 $\triangle A E C 2$ mice with PCR (Figure S6A). The Zip8 $\triangle A E C 2$ mice developed normally without obvious physical defect. There are no significant changes in lung histology between 12 weeks old adult Zip8 $\triangle{ }_{\triangle A E C 2}$ mice and littermates (Figure S6B).

We assessed AEC2s in the lungs of Zip8 $\triangle A E C 2$ mice and littermate control two weeks after 4 doses of tamoxifen of $200 \mathrm{mg} / \mathrm{kg}$. The ZIP8+/Tomato+ cells were significantly reduced in the lung of Zip8 $\triangle A E C 2$ mice (Figure 7A, 7C). AEC2s with Zip8 deletion showed decreased intracellular zinc levels compared to that of AEC2s from control mice (Figure 7B). The total number of AEC2s recovered from uninjured lungs was not significantly different between Zip8 $\triangle A E C 2$ mice and littermate controls (Figure 7D). However, AEC2s isolated from uninjured Zip8 $\triangle A E C 2$ mice had reduced Sirt1 expression (Figure 7E) and lower CFEs with $3 \mathrm{D}$ organoid culture than that of $\mathrm{AEC} 2 \mathrm{~s}$ from littermate controls (Figure $7 F)$.

Next we treated 8-10 weeks old young adult Zip8 $\triangle A E C 2$ mice and littermate controls with $2.5 \mathrm{U} / \mathrm{kg}$ bleomycin following 4 doses of tamoxifen injection and sacrificed the mice at day 4 after bleomycin treatment. The AEC2s recovered from each lung of bleomycin injured Zip8 $\triangle A E C 2$ mice was much fewer than the cells from littermate controls (Figure 7G).

AEC2s from bleomycin injured Zip8 $\triangle A E C 2$ mouse lungs showed severe regenerative defect with 3D organoid culture and they gave rise to extremely fewer and smaller colonies compared to AEC2s from littermate controls (Figure 7H,7l). AEC2s derived from 3D organoids of AEC2s from Zip8 $\triangle A E C 2$ mice showed reduced percentage of Ki67+Tomato+ cells (Figure $7 \mathrm{~J}$ ) and dramatic decrease of Pdpn expression (Figure 7K).

\section{Zinc metabolism regulated lung fibrosis}


370 Next we investigated the role of zinc metabolism in regulating lung fibrosis. We treated Zip8 $\delta A E C 2$ mice and control mice with $2.5 \mathrm{U} / \mathrm{kg}$ bleomycin after tamoxifen injection and harvested the lungs 21 days after bleomycin injury for fibrosis assessment using hydroxyproline assay. Lungs of bleomycin injured Zip8_AEC2 mice showed increased hydroxyproline contents compared to that of the lungs from control mice (Figure $7 \mathrm{~L}, 7 \mathrm{M}$ ).

375 To further confirm that zinc deficiency worsened lung fibrosis, we applied bleomycin lung injury model to the mice fed with modified zinc diet. We fed the wild type mice with zinc deficient diet (zinc 20 PPM) and control diet (zinc 80 PPM) for three weeks before bleomycin injury $(2.5 \mathrm{U} / \mathrm{kg})$ and the mice were kept on the same diet until end of the experiments. We harvested lung tissues at day 21 after bleomycin treatment for fibrosis assessment. Mice fed with zinc deficient diet developed worse lung fibrosis showing as increased hydroxyproline contents compared to the lungs of mice fed with control diet (Figure 7N, 7O). These findings demonstrate that zinc transporter ZIP8 and zinc metabolism have a key role in regulating alveolar progenitor renewal. Reduced zinc transporter ZIP8 of AEC2s in aging and in IPF contributes to impaired alveolar repair and lead to lung fibrosis. 


\section{Discussion}

Aging have been recognized as an important risk factor for IPF. Evidences indicates that the main hallmarks of aging occur prematurely in IPF and primarily affect epithelial cells (Rojas et al., 2015; Selman and Pardo, 2014; Thannickal, 2013). However, the molecular mechanism that regulates AEC2 progenitor cell renewal and lung fibrosis during aging and in IPF is poorly understood. In this study we identified reduced expression of SLC39A8, the gene encoding zinc transporter ZIP8 in AEC2s from lungs of patients with 395 IPF using single cell transcriptome analysis. We further observed reduced level of cell surface ZIP8 expression and decreased intracellular zinc of IPF AEC2s. Most importantly we showed that ZIP8 plays an important role in regulating AEC2 progenitor renewal and ZIP8neg AEC2s had reduced renewal capacity relative to that of ZIP8pos AEC2s. Zinc sulfate treatment was able to promote renewal of AEC2s from both healthy and IPF lungs.

400 This is the first evidence to show that zinc metabolism homeostasis is crucial for maintaining progenitor function of AEC2s and there is a defect of zinc metabolism with IPF AEC2s.

Our findings with AEC2s from human diseased lung were corroborated well by the results from animal model study in scRNA-seq, in vitro culture, as well as in vivo lung 405 fibrosis. With animal model of aging, we found that the ZIP8 expression and renewal capacity of $A E C 2 s$ from lungs of old mice were reduced relative to that of $A E C 2 s$ from young mice. Zinc treatment promoted renewal of AEC2s from young mice while the response to zinc diminished with the AEC2s from older mice. These data demonstrate that decreased ZIP8 expression was associated with aging and that contributed to the

410 impaired renewal capacity of AEC2s. Mice with targeted deletion of ZIP8 in AEC2 compartment had reduced AEC2 renewal and developed more severe lung fibrosis further confirmed the role of ZIP8 in regulating AEC2 progenitor function and lung fibrosis.

There are multiple factors that might contribute to the impaired progenitor function of AEC2s during aging including apoptosis (Korfei et al., 2008), ER stress (Lawson et al., 415 2011), senescence (Minagawa et al., 2011), and mitochondria dysfunction (Bueno et al., 2015). ZIP8 deficiency added another critical player that contributes to the debilitated progenitor function of AEC2s in the aged lung. Zinc deficiency in elderly has been reported (Bogden, 2004; Prasad et al., 1993). Zinc metabolism may have several ways to influence alveolar progenitor activities. One of the obvious mechanisms is through the 420 zinc-dependent sirtuin signaling. We observed down regulation of sirtuin signaling with IPF AEC2s relative to AEC2s from healthy lungs. With 3D Matrigel organoid culture, we showed that sirtuin activator enhanced and sirtuin inhibitor suppressed colony formation of AEC2s. These data indicated that sirtuin signaling is crucial for AEC2 progenitor renewal through a zinc-dependent mechanism. We further showed that ZIP8neg AEC2s had lower sirt1 expression compared to ZIP8pos cells and zinc treatment elevated sirt1 
expression as well as renewal capacity of AEC2s. We concluded that ZIP8 and zinc metabolism regulate AEC2 progenitor function through sirtuin signaling.

In addition to the sirtuin signaling, zinc may have a role in alveolar progenitor and in lung repair through other mechanisms such as regulating antioxidative stress (Tan et al., 2018) and expression of glutathiones (Jiang et al., 1998). We observed a downregulation of NRF2-mediated antioxidative stress as well as glutathione redox response in IPF AEC2s. Furthermore, we have reported previously that loss cell surface hyaluronan (HA) and insufficient IL-6 production contributed to the impaired progenitor function of AEC2s in IPF lungs (Liang et al., 2016). We have examined the correlation between ZIP8 expression and HA in AEC2s. We found that ZIP8 expression is positively correlated with HA levels in AEC2s. ZIP8pos AEC2s showed higher HA level than that of ZIP8neg cells. Zinc treatment increased AEC2 cell surface HA along with ZIP8 and SIRT1 expression. Our data suggest that HA and ZIP8 are both important for AEC2 renewal and loss of both molecules with IPF AEC2s might due to loss epithelial integrity of the cells.

440 Further study is needed to investigate the interaction between zinc metabolism and HA production of AEC2s.

How are aging, zinc metabolism, alveolar progenitor, and fibrosis interconnected? We think that the accumulated events in genetic and epigenetic reprogramming during aging may lead to many key changes such as zinc metabolic dysregulation. Many transcription factors (Liu et al., 2013; Prasad, 2008) and epigenetic mediators such as histone deacetylases (HDACs) (Blanquart et al., 2019; Ren et al., 2017; Seto and Yoshida, 2014) are zinc-dependent. For example, ZIP8 is regulated by NFкB (Liu et al., 2013), whereas zinc regulates NFKB activity (Vasto et al., 2007). Both HDACs and histone H1 polypeptides are the substrates of sirtuins (Seto and Yoshida, 2014; Vaquero et al., 2004).

450 Thus, the zinc defect would further fuel the dysregulation of genetic and epigenetic reprogramming. These compounding factors would further deteriorate the alveolar progenitor, leading to the loss of alveolar epithelial integrity and proper repair after injury.

In summary, we have uncovered a novel mechanism that ZIP8-Sirtuin axis regulates AEC2 renewal. Loss of ZIP8 in AEC2s during aging and in IPF results in disrupted sirtuin function, impaired AEC2 progenitor function, and lung fibrosis. Target zinc metabolism may be a new avenue for developing therapeutics for lung fibrosis. 


\section{Experiment procedures}

\section{Study with cells from lung tissues of human subjects}

460 The use of human tissues for research were approved by the Cedars-Sinai Medical Center Institutional Review Board (Pro00032727), and UCLA Institutional Review Board (13-000462-AM-00019). Informed consent was obtained from each subject.

\section{Mice}

465 All animal experiments were approved by the Institutional Animal Care and Use Committee at Cedars-Sinai Medical Center (IACUC008529). All mice were housed in a pathogen-free facility at Cedars-Sinai Medical Center. SFTPC-CreER mice and RosaTomatoflox/flox mice were described previously (Barkauskas et al., 2013; Liang et al., 2016). Slc39a8flox/+ was generated using the Cre/loxP system and acquired from MMRRC 470 Repository. All mice have been backcrossed on C57BI/6J background more than six generations. Eight to 12 weeks old (young) and 18 months old (aged) wild-type C57BI/6J mice were obtained from The Jackson Laboratory and housed in the institution facility at least 2 weeks before experiments. Animals were randomly assigned to treatment groups, and they were age- and sex-matched.

\section{Bleomycin instillation}

Bleomycin instillation was described previously (Liang et al., 2016). Under anesthesia, the trachea was surgically exposed. $1.25-2.5 \mathrm{U} / \mathrm{kg}$ bleomycin (Hospira, Lake Forest, IL) in $25 \mu \mathrm{l}$ PBS was instilled into the mouse trachea with a $25-\mathrm{G}$ needle inserted between the cartilaginous rings of the trachea. Control animals received saline alone. The

480 tracheostomy site was sutured, and the animals were allowed to recover. Mice were sacrificed at different time points and lung tissue were collected for experiments.

\section{Hydroxyproline}

Collagen contents in mouse lungs were measured with a conventional hydroxyproline method (Jiang et al., 2004; Liang et al., 2019). In brief, lung tissues were vacuum dried and hydrolyzed with $6 \mathrm{~N}$ hydrochloride acid at $120 \circ \mathrm{C}$ for overnight. Hydroxyproline content was measured and expressed as mg per lung. The ability of the assay to completely hydrolyze and recover hydroxyproline from collagen was confirmed using samples containing known amounts of purified collagen.

\section{Lung tissue and histology}


490 Mice were sacrificed at various time points with or without bleomycin treatment under anesthesia. The trachea was cannulated, and the lung was inflated with $1.0 \mathrm{~mL}$ of $10 \%$ neutral buffered formalin. Mouse lung tissue was fixed, embedded in paraffin, sectioned to $5 \mu \mathrm{m}$ slices for H\&E and and Masson's trichrome staining (Geng et al., 2019; Jiang et al., 2004; Liang et al., 2019).

\section{Cell lines}

Mouse lung fibroblast cell line, MLg2908 (Catalog CCL-206), was from ATCC (Manassas, VA). Mycoplasma contamination was assessed with a MycoFluor ${ }^{\mathrm{TM}}$ Mycoplasma Detection Kit (Catalog M7006, Thermo Fisher Scientific) and cells used for experiments were free of mycoplasma contamination.

\section{RNA analysis}

RNA was extracted from mouse AEC2s or human AEC2s using TRIzol Reagent. For realtime PCR analysis, $0.5 \mu \mathrm{g}$ total RNA was used for reverse transcription with the High Capacity cDNA Reverse Transcription Kit (Applied Biosystems, Carlsbad, California). One microliter cDNA was subjected to real-time PCR by using Power SYBR Green PCR Master Mix (Applied Biosystems) and the ABI 7500 fast Real-Time PCR system (Applied Biosystems). The specific primers were designed on the basis of cDNA sequences deposited in the GenBank database: Human SLC39A8 (NM_001135146) forward CATCTGTCCAGCAGTCTTACAGC, and reverse GACAGGAATCCATATCCCCAAACT. Mouse SIc39a8 (NM_001135149): forward AGCGATCCTGTGTGAGGAGT and reverse CGGAGAGGAAGTTGAACAGC. Human SFTPC (NM_003018.4): forward ATCCCCAGTCTTGAGGCTCT and reverse CTTCCACTGACCCTGCTCAC. Mouse Sftpc (NM_011359.2): forward GCAGGTCCCAGGAGCCAGTTC and reverse GGAGCTGGCTTATAG GCCGTCAG. Human PDPN (NM_006474.5): forward GGAAGGTGTCAGCTCTGCTC and reverse CGCCTTCCAAACCTGTAGTC. Mouse

515 Pdpn (NM_010329.3): forward GCACCTCTGGTACCAACGCAGA and reverse TCTGAGGTTGCTGAGGTGGACAGT. Human SIRT1 (NM_012238.5): forward TGCCGGAAACAATACCTCCA and reverse AGACACCCCAGCTCCAGTTA. Mouse Sirt1 (NM_019812.3): forward GAGCTGGGGTTTCTGTCTCC and reverse CTGCAACCTGCTCCAAGGTA. Human GAPDH (NM_002046) forward, 520 CCCATGTTCGTCATGGGTGT and reverse, TGGTCATGAGTCCTTCCACGATA. Mouse Gapdh (NM_00100130): forward ATCATCTCCGCCCCTTCTG and reverse, GGTCATGAGCCCTTCCACAAC. The relative expression level of each gene was determined against GAPDH level in the same sample. The fold-change of the target genes was calculated by using the $2-\Delta \Delta \mathrm{c}$ T method. 


\section{Mouse lung dissociation and flow cytometry}

Mouse lung single cell suspensions were isolated as previously described (Liang et al., 2016). In brief, lungs were perfused with $10 \mathrm{ml}$ PBS and then digested with $4 \mathrm{U} / \mathrm{ml}$ elastase (Worthington Biochemical Corporation, NJ) and $100 \mathrm{U} / \mathrm{ml}$ DNase I (Sigma), and

530 resuspended in Hanks' balanced saline solution supplemented with $2 \%$ fetal bovine serum (FBS), $10 \mathrm{mM}$ HEPES, $0.1 \mathrm{mM}$ EDTA (HBSS+ buffer). The cell suspension was incubated with primary antibodies including CD24-PE, EpCAM-PE-Cy7, Sca-1-APC, biotinylated-CD31, -CD34, and -CD45, as well as conjugated ZIP8 (rabbit IgG) for 45 minutes. Biotin-conjugated antibodies were detected following incubation with streptavidin-APC-Cy7 (catolog 405208, BioLegend, San Diego, CA), while ZIP8 was detected with goat anti rabbit IgG-FITC for 30 minutes. Dead cells were discriminated by 7-amino-actinomycin D (7-AAD) (BD Biosciences, San Diego, CA) staining. Flow cytometry was performed using a Fortesa flow cytometer and FACSAria III sorter (BD Immunocytometry Systems, San Jose, CA) and analyzed using Flow Jo 9.9.6 software 540 (Tree Star, Ashland, OR).

Primary antibodies EpCAM-PE-Cy7 (clone G8.8, Catalog \# 118216, RRID $A B \_1236471$ ) and Ki-67 (clone 16A8, catalog \# 652403, AB_2561524) were from BioLegend. CD24-PE (clone M1/69, Catalog \# 12-0242-82, RRID AB_467169), Sca-1 (Ly-6A/E)-APC (clone D7, Catalog \# 17-5981-82, RRID AB_469487), CD31 (PECAM-1)

545 (clone 390, Catalog \# 13-0311-85, RRID AB_466421), CD34 (clone RAM34, Catalog \# 13-0341-85, RRID AB_466425), and CD45 (clone 30-F11, Catalog \# 13-0451-85, RRID AB_466447) were all from eBioscience (San Diego, CA). SLC39A8 polyclonal antibody (Catalog \# PA5-26368, RRID AB_2543868) was from ThermoFisher.

\section{Human lung dissociation and flow cytometry}

Human lung single cell isolation and flow cytometer analysis were performed as described previously (Barkauskas et al., 2013; Liang et al., 2016). In brief, human lung tissues were minced and then digested with $2 \mathrm{mg} / \mathrm{ml}$ dispase II, followed by $10 \mathrm{U} / \mathrm{ml}$ elastase and 100 $\mathrm{U} / \mathrm{ml}$ DNase I digestion. Finally, cells were filtered through $100 \mu \mathrm{m}$ cell strainer, and lysed with red blood cell lysis to get single cell suspension. Antibody staining was similar as with mouse cells. Flow cytometry was performed with Fortesa and FACSAria III flow cytometer and analyzed with Flow Jo 9.9.6 software. Anti-human CD31 (clone WM59, Catalog \# 303118, RRID AB_2247932), CD45 (clone WI30, Catalog \# 304016, RRID AB_314404), EpCAM (clone 9C4, Catalog \# 324212, RRID AB_756086) were from

560 BioLegend. SLC39A8 polyclonal antibody (Catalog \# PA5-26368, RRID AB_2543868) and goat anti-mouse IgG/lgM (Catalog \# A-10680, RRID AB_2534062) were from ThermoFisher. HTII-280 (Gonzalez et al., 2010) was a gift from Dr. L. Dobbs lab at UCSF. 


\section{Intracellular staining for zinc of human and mouse AEC2s}

Zinc assay kit (catalog \# ab241014) was from Abcam (Cambridge, MA). Single cells isolated from human and mouse lungs were cultured on collagen IV coated plated with $100 \mu \mathrm{m} \mathrm{ZnSO} 4$ for overnight. Cells were first stained for intracellular zinc following manufacturer's protocol, then AEC2 cell surface markers were stained. Flow cytometry was performed using a Fortesa flow cytometer. Intracellular zinc staining of gated human or mouse AEC2s was analyzed using Flow Jo 9.9.6 software (Tree Star, Ashland, OR).

\section{Matrigel culture of human and mouse AEC2s}

Flow sorted human (EpCAM+HTII-280+) or mouse (EpCAM+CD24-Sca-1-) AEC2s were cultured in Matrigel/medium (1:1) mixture in presence of lung fibroblasts MLg2908 cells (Barkauskas et al., 2013; Chen et al., 2012a; Liang et al., 2016). $100 \mu \mathrm{l} \mathrm{Matrigel/medium}$ mix containing $3 \times 10_{3}$ AEC2s and $2 \times 10_{5}$ MLg2908 cells (Catalog CCL-206, ATCC,

575 Manassas, VA) were plated into each 24 well $0.4 \mathrm{~mm}$ Transwell inserts. $400 \mu$ of medium were added in the lower chambers. Cells were cultured with medium alone or with the treatments indicated. Medium was described previously (Liang et al., 2016). Matrigel (growth factor reduced basement membrane matrix) (catalog \# 354230) was from Corning Life Sciences (Tewksbury, MA). For cell treatment, the following chemicals were 580 used. $100 \mu \mathrm{M} Z n S O 4,1 \mathrm{mM}$ TPEN, , and $150 \mathrm{mM}$ splitomicin were from Sigma (St. Louis, MO). $1 \mathrm{mM}$ SRT1720 was from Selleck Chemicals (Houston, TX). Same volume of DMSO was used as control. Fresh medium was changed every other day. Cultures were maintained in humidified $37 \circ \mathrm{C}$ and $5 \% \mathrm{CO}_{2}$ incubator. Colonies were visualized with a Zeiss Axiovert40 inverted fluorescent microscope (Carl Zeiss AG, Oberkochen, 585 Germany). Number of colonies with a diameter of $\geqslant 50 \mu \mathrm{m}$ from each insert was counted and colony-forming efficiency (CFE) was determined by the number of colonies in each culture as a percentage of input epithelial cells at 12 day after plating (dpp).

\section{Organoid size measurement}

Organoids derived from flow sorted Rosa-Tomato + cells from SFTPC-CreER+;Rosa590 Tomatofloxflox mice and SFTPC-CreER+;Rosa-Tomatofloxfflox ;Slc39a8floxfflox (Zip8_AEC2) mice were pictured at day 12 after plating using Zeiss Axiovert40 inverted fluorescent microscope. ZEN pro 2012 software (Zeiss) was used for measure surface area of the pictured organoids same as previously described (Liang et al., 2016).

\section{scRNA-seq}

595 scRNA-sequencing was performed in Cedars-Sinai Medical Center Genomic Core. In brief, flow sorted human and single cells were lysed, mRNA was reverse transcribed and amplified as previously described (Xie et al., 2018). Barcoding and library preparation 
were done with standard procedures according to manufacture manuals (10X Genomics, Pleasanton, CA, USA). The barcoded libraries were sequenced with NextSeq500 600 (Illumina, San Diego, CA, USA) to obtain a sequencing depth of $\sim 200 \mathrm{~K}$ reads per cell.

Raw scRNA-seq data was aligned to human genome GRCh38 and mouse genome mm10 with Cell Ranger (10X Genomics) respectively. Downstream quality control, normalization and visualization were done with Seurat package. For quality control, the output expression matrix from Cell Ranger was done based on number of genes detected 605 in each cell, number of transcripts detected in each cell and percentage of mitochondrial genes. The expression matrix was then normalized and visualized with UMAP.

Ingenuity Pathway Analysis (IPA) was performed as described previously (Kramer et al., 2014) . Differential expression genes with logFC over 0.1 between healthy and IPF $A E C 2 s$ were analyzed with $R$ software. The data was then imported into IPA software

610 (Qiagen, Hilden, Germany). Canonical pathways from the core analyze were further analyzed.

\section{Statistics}

The statistical difference between groups in the bioinformatics analysis was calculated using the Wilcoxon Signed-rank test. For the scRNA-seq data the lowest p-value 615 calculated in Seurat was $p<2.2 e-10-16$. For all other data the statistical difference between groups was calculated using Prism (version 8.4.3) (GraphPad Software, San Diego, CA) and the exact value was shown. Data are expressed as the mean \pm SEM. All experiments were repeated two or more times. Data were normally distributed and the variance between groups was not significantly different. Differences in measured variables between experimental and control group were assessed using Student's t-tests. One-way followed by Bonferroni's or two-way ANOVA followed Tukey's multiple comparison test was used for multiple comparisons. Results were considered statistically significant at $p<0.05$. 


\section{Acknowledgments}

The authors thank the members of our laboratory for support and helpful discussion during the course of the study. This work was supported by National Institutes of Health grants R35-HL150829, R01-HL060539, R01-Al052201, R01-HL077291 (PWN), and R01HL122068 (DJ and PWN), and P01-HL108793 (PWN and DJ). SCR was supported by 630 European Union's Horizon 2020 research and innovation programme under the Marie Skłodowska-Curie grant agreement 797209. We thank Dr. L. Dobbs of UCSF providing antibodies for the study.

Conflict of Interest: The authors declare that there is no conflict of interest.

\section{Author Contributions}

$635 \mathrm{JL}, \mathrm{DJ}$, and PWN conceived the study. JL performed most of the experiments and analyzed the data. GH analyzed single cell RNA transcriptome data, performed flow cytometry analysis, and prepared figures. JL, GH, XL, YW, ND, CY, and DJ analyzed single cell RNA transcriptome data. $X L, F T, N L, A B, S R$, and TX took part in mouse, cell culture, and biological experiments. SSW and JB provided human samples and

640 interpreted data. BS and WCP interpreted data and contributed with comments on the manuscript. JL, DJ, and PWN wrote the paper. All authors read and reviewed the manuscript.

\section{Lead Contact Statement}

Further information and requests for resources and reagents should be directed to and will be fulfilled by the Lead Contact, Paul Noble (paul.noble@cshs.org).

\section{Materials Availability}

All unique/stable reagents generated in this study are available from the Lead Contact with a completed Materials Transfer Agreement.

\section{Data and Code Availability}

The deposition of the raw data files of the single cell RNA-seq are in progress. The accession numbers for the the RNA-seq analyses and the dataset information will be updated accordingly. $R$ code files used for data integration and analysis are available at https://github.com/jiang-fibrosis-lab. 


\section{Figure legend}

Figure 1. scRNA-seq identifies dysregulation of metabolism and stem cell signaling in IPF AEC2s

(A) UMAP plots of flow enriched EpCAM+CD31-CD45- cells from healthy $(11,381$ cells, $n$ $660=6)$ and IPF lungs $(14,687$ cells, $n=6)$.

(B) Distribution of epithelial cell types.

(C) Expression of AEC2 marker genes from healthy and IPF lungs.

(D) SFTPC expression in healthy and IPF AEC2s by RT-PCR ( $n=6$ and $\left.4,{ }^{*} \mathrm{p}<0.05\right)$.

(E) IPA pathway analysis of AEC2s from healthy and IPF lungs.

665 (F) Sirtuin activation score of healthy and IPF AEC2s.

(G) 3D organoid culture of AEC2s from healthy lungs treated with SRT1720 and splitomicin $\left(n=4-5,{ }^{* * *} p<0.001,{ }^{* * * *} p<0.0001\right)$.

$(\mathrm{H}, \mathrm{I})$ SIRT1 expression in fresh isolated AEC2s $(\mathrm{H})$ and AEC2s derived from 3D organoids (I) by qPCR $\left(n=4-6,{ }^{*} p<0.05\right)$.

Figure 2. Dysregulation of zinc metabolism of IPF AEC2s.

(A) scRNA-seq own regulated zinc metabolism related genes in IPF AEC2s.

(B) SLC39A8 expression of human lung epithelial cell types.

(C) SLC39A8 expression of AEC2s from healthy and IPF lungs.

675 (E) SLC39A8 expression of AEC2s freshly isolated from lung tissues $\left(n=4\right.$ each. ${ }^{* * *} \mathrm{p}<$ $0.001)$.

(E) SLC39A8 expression of AEC2s derived from 3D cultured organoids $\left(n=7-8 .{ }^{* \star * *} p\right.$ $<0.0001)$.

$(F, G)$ Flow cytometry of cell surface ZIP8 level and percent of ZIP8+ cells of healthy and 680 IPF AEC2s. $\left(n=7-11,{ }^{* \star * *} \mathrm{p}<0.0001\right)$.

(H) SLC39A8 expression of healthy and ILD lung tissues $\left(n=137-255,{ }^{* * *} p<0.0001\right)$.

(I) Representative plots of intracellular zinc staining of human AEC2s.

Figure 3. ZIP8 expression is associated with AEC2 renewal.

685 (A) Flow cytometry plots of gated ZIP8+ and ZIP8- AEC2s.

(B) CFE of flow sorted ZIP8+ and ZIP8- AEC2s from healthy and IPF lungs ( $n=4-6$, $\left.{ }^{* * *} p<0.001,{ }^{* * *} p<0.0001\right)$.

(C, D) SIRT1 (C, $\mathrm{n}=5$ each, $\left.{ }^{*} \mathrm{p}<0.05\right)$.) and PDPN ( $\mathrm{D}, \mathrm{n}=3$ each, $\left.{ }^{*} \mathrm{p}<0.05\right)$.) expression of AEC2s derived from 3D cultured organoids of ZIP8+ and ZIP8- AEC2s from healthy 690 lung.

(E) CFE of AEC2s from healthy and IPF lungs with and without zinc sulfate $(100 \mu \mathrm{M})$ treatment $\left(n=5-7,{ }^{* * * *} p<0.0001\right)$. 
(F) CFE of ZIP8+ and ZIP8- AEC2s with and without zinc sulfate $(100 \mu M)$ treatment $(n=$ 3 each. ${ }^{* * * *} p<0.0001$; ns, not significant).

695 (G) CFE of AEC2s with and without zinc sulfate and TPEN treatment $\left(n=3-5,{ }^{* *} p<\right.$ $\left.0.01,{ }^{* * * *} \mathrm{p}<0.0001\right)$.

(H-J) Expression of SLC39A8 ( $\left.\mathrm{n}=4-6,{ }^{* *} \mathrm{p}<0.001\right), \operatorname{SIRT1}\left(\mathrm{n}=3-4,{ }^{*} \mathrm{p}<0.05\right)$ and SFTPC $\left(n=4-5,{ }^{*} p<0.05\right)$ in AEC2s with and without zinc sulfate by RT-PCR (ns, not significant).

Figure 4. Decreased ZIP8 expression and renewal capacity of aging AEC2s.

(A) Gating strategy for ZIP8 expressing AEC2s from young and old mice by flow cytometry.

(B) Number of total cells recovered from young and old mouse lungs $(n=5-6)$.

$(C, D)$ Percent and number of EpCAM+CD31-CD34-CD45- epithelial cells (R1 in panel A) $705 \quad\left(n=5-6 .{ }^{* *} p<0.01,{ }^{* * * *} p<0.0001\right)$.

(E) Percent of CD24-Sca-1- AEC2s (R2 in panel $A$ ) within total lung epithelial cells (R1 in panel A) $\left(n=4-6,{ }^{* *} p<0.01\right)$.

(F) Number of AEC2s were recovered from young and old mouse lung $\left(n=6-7,{ }^{* * *} p<\right.$ 0.0001).

710 (G) Percent of ZIP8+ cells within total AEC2s (R3 in panel A) $\left(n=5-6,{ }^{* *} p<0.01\right)$.

(H) Number of ZIP8+ cells were recovered from young and old mouse lung $\left(n=5-6,{ }^{* *} p\right.$ $<0.01$ ).

(I) CEF of mouse AEC2s isolated from young and old mouse lungs $\left(n=6-7,{ }^{* *} p<0.01\right)$. $(\mathrm{J}, \mathrm{K})$ Expression of Sftpc $(\mathrm{J})\left(\mathrm{n}=3-4,{ }^{*} \mathrm{p}<0.05\right)$ and Pdpn $(\mathrm{K})\left(\mathrm{n}=6\right.$ each, $\left.{ }^{* *} \mathrm{p}<0.01\right)$ of mouse AEC2s derived from 3D cultured organoids by RT-PCR.

Figure 5. Decreased ZIP8 expression and renewal capacity of aging AEC2s.

(A) IPA pathway analysis of AEC2s from young and aged mice 4 days after bleomycin.

(B) qPCR SIc39a8 expression of AEC2s from uninjured mouse lungs ( $n=6-7,{ }^{* *} p<$ 720 0.01).

(C, D) Expression of Slc39a8 (C) and AEC2 marker genes Sftpc and Abca3 (D) of AEC2s from day 0 and bleomycin day 4, day 14 young and old mouse lungs.

(E) Number of AEC2s were recovered from mouse lung tissue at day 14 after bleomycin injury $\left(n=3-6,{ }^{* *} p<0.001\right)$.

Figure 6. Aging blunted the response of AEC2s to exogenous zinc.

(A) CFE of AEC2s from uninjured wild type mice treated with exogenous $\mathrm{ZnSO} 4(100 \mu \mathrm{M})$ $\left(n=6,{ }^{* *} p<0.01\right)$.

(B) Flow cytometry gated ZIP8 + AEC2s cultured with medium only or medium containing $730 \quad 100 \mu \mathrm{M}$ ZnSO4.

(C) Percent of ZIP8+ AEC2s $\left(n=6,{ }^{* * *} p<0.001\right)$. 
(D) CFE of mouse AEC2s isolated from lungs of 2.5, 12, 14, and 18-month-old mice $(\mathrm{n}=$ $\left.3-4,{ }^{*} p<0.05,{ }^{* *} p<0.01,{ }^{* * *} p<0.001,{ }^{* * * *} p<0.0001\right)$.

(E-G) qPCR gene expression of Slc39a8 (D), Sftpc (E), and Pdpn (F) ( $\mathrm{n}=3-4,{ }^{*} \mathrm{p}<0.05$, $\left.{ }^{* *} p<0.01,{ }^{* * *} p<0.001,{ }^{* * *} p<0.0001\right)$.

Figure 7. Targeted deletion of SIc39a8 decreased AEC2 renewal and worsened lung fibrosis

(A) ZIP8 expressing of AEC2s from Zip8 $\triangle \mathrm{AEC2}$ and control mice by flow cytometry.

(B) Intracellular zinc in AEC2s from Zip8 $\triangle A E C 2$ (mean 25.03\%, $n=4$ ) and control mice (mean $36.10 \%, n=6)$ by flow cytometry $(p<0.01)$.

(C) ZIP8 expressing AEC2s (Tomato+) from Zip8 $\triangle A E C 2$ and control mice by flow cytometry $\left(n=5\right.$ each, $\left.{ }^{* * *} p<0.0001\right)$.

(D) Number of AEC2s recovered from uninjured lungs of Zip8 $\triangle A E C 2$ and control mice $(n=$ $7456-8)$.

(E) Sirt1 expression of AEC2s by qPCR $\left(n=4,{ }^{*} p<0.05\right)$.

(F) CFE of AEC2s from uninjured mouse lungs $\left(n=6\right.$ each, $\left.{ }^{* *} p<0.01\right)$.

(G) Number of AEC2 recovered per lung of Zip8 ${ }_{\triangle A E C 2}$ and control mice at day 4 after bleomycin $\left(n=5\right.$ each, $\left.{ }^{* *} p<0.01\right)$.

$750(\mathrm{H}, \mathrm{I})$ CFE $(n=5-6)$ and colony size $(n=28-86)$ of AEC2s isolated from Zip8 $\triangle A E C 2$ and control mice at day 4 after bleomycin $\left({ }^{* * *} \mathrm{p}<0.001,{ }^{\star \star * *} \mathrm{p}<0.0001\right)$.

(J) Ki67 expression of AEC2s derived from 3D cultured organoids of AEC2s from bleomycin injured Zip8 $\triangle A E C 2$ and control mice by flow cytometry $\left(n=6\right.$ each, ${ }^{* *} p<0.05$ ).

(K) Pdpn expression of AEC2s derived from 3D cultured organoids of AEC2s from

755 bleomycin injured Zip8 $\triangle A E C 2$ and control mice by qPCR $\left(n=5\right.$ each, $\left.{ }^{* \star *} \mathrm{p}<0.0001\right)$.

(L) Experiment layout of Zip8 ${ }_{\triangle \mathrm{AEC}}$ and control mice treated with $1.25 \mathrm{U} / \mathrm{kg}$ bleomycin following tamoxifen injection.

(M) Hydroxyproline levels of lungs from Zip8 $\triangle A E C 2$ and control mice harvested at day 21 after bleomycin $\left(n=5-7,{ }^{*} p<0.05\right)$.

760 (N) Experiment layout of wild-type mice treated with $2.5 \mathrm{U} / \mathrm{kg}$ bleomycin following zinc deficient and control diet.

(O) Hydroxyproline levels of lungs of mice fed with zinc deficient diet and control diet harvested at day 21 after bleomycin $\left(n=8-10,{ }^{*} p<0.05\right)$. 
Figure S1. scRNA-seq analysis of lung epithelial cells.

(A) UMAP plots of lung epithelial cells from IPF and healthy donors ( $n=6$ each).

(B) Feature plots of lung epithelial clusters grouped by subject.

(C) Feature plots of lung epithelial clusters by classic cell type markers.

Figure S2. Effect of SIRT activator and inhibitor on mouse AEC2s.

$(A, B)$ CFE of AEC2 from uninjured wild type mice treated with SRT $1720(A)$, splitomicin (B) with DMSO as control $\left(n=3\right.$ each. $\left.{ }^{* * *} p<0.001\right)$.

Figure S3. Specific expression of SLC39A8 in alveolar epithelial cells.

(A) Expression of SLC39A1-14 in lung tissue.

(B) Expression of SLC39A8 in human tissues and organs.

(C) Expression of $S \angle C 39 A 8$ in AEC1s from healthy and IPF lungs.

780 (D) Expression of SLC39A1 and SLC39A7 in AEC2s from healthy and IPF lungs.

(E) Expression of ZNT family genes SLC30A1, SLC30A5, SLC30A6, SLC30A7, and SLC30A9 in AEC2s from healthy and IPF lungs.

(F) Expression of S/c39a8 in mouse lung epithelial cells.

Figure S4. Correlation of ZIP8 and hyaluronan expression.

(A) Percent of HABP+ of ZIP8neg and ZIP8pos ACE2s by flow cytometry.

(B) Percent of $\mathrm{HABP}+\mathrm{AEC2s}$ with and without $100 \mu \mathrm{m}$ ZnSO4 treatment.

Figure S5. No obvious morphological defect in Zip8 deletion mice.

(A) A representative genotyping result of Sftpc-CreER+;RosaTomato+;Zip8floxfllox (Zip8 $\triangle A E C 2)$ mice.

$(B, C)$ Histological characterization ( $B, H \& E$ staining; $C$, trichrome) of uninjured lungs of Zip8 $\triangle A E C 2$ and control mice at $8-10$ weeks old. 


\section{REFERENCES}

Adams, T.S., Schupp, J.C., Poli, S., Ayaub, E.A., Neumark, N., Ahangari, F., Chu, S.G., Raby, B.A., Deluliis, G., Januszyk, M., Duan, Q., Arnett, H.A., Siddiqui, A., Washko, G.R., Homer, R., Yan, X., Rosas, I.O., and Kaminski, N. (2020). Single-cell RNAseq reveals ectopic and aberrant lung-resident cell populations in idiopathic pulmonary fibrosis. Science Advances 6, eaba1983.

Alder, J.K., Chen, J.J., Lancaster, L., Danoff, S., Su, S.C., Cogan, J.D., Vulto, I., Xie, M., Qi, X., Tuder, R.M., Phillips, J.A., 3rd, Lansdorp, P.M., Loyd, J.E., and Armanios, M.Y. (2008). Short telomeres are a risk factor for idiopathic pulmonary fibrosis. Proc Natl Acad Sci U S A 105, 13051-13056.

Anathy, V., Lahue, K.G., Chapman, D.G., Chia, S.B., Casey, D.T., Aboushousha, R., van der Velden, J.L.J., Elko, E., Hoffman, S.M., McMillan, D.H., Jones, J.T., Nolin, J.D., Abdalla, S., Schneider, R., Seward, D.J., Roberson, E.C., Liptak, M.D., Cousins, M.E., Butnor, K.J., Taatjes, D.J., Budd, R.C., Irvin, C.G., Ho, Y.S., Hakem, R., Brown, K.K., Matsui, R., Bachschmid, M.M., Gomez, J.L., Kaminski, N., van der Vliet, A., and Janssen-Heininger, Y.M.W. (2018). Reducing protein oxidation reverses lung fibrosis. Nat Med 24, 1128-1135.

Barkauskas, C.E., Cronce, M.J., Rackley, C.R., Bowie, E.J., Keene, D.R., Stripp, B.R., Randell, S.H., Noble, P.W., and Hogan, B.L. (2013). Type 2 alveolar cells are stem cells in adult lung. The Journal of clinical investigation 123, 3025-3036.

Blanquart, C., Linot, C., Cartron, P.F., Tomaselli, D., Mai, A., and Bertrand, P. (2019). Epigenetic Metalloenzymes. Curr Med Chem 26, 2748-2785.

Bogden, J.D. (2004). Influence of zinc on immunity in the elderly. J Nutr Health Aging 8 , 48-54.

820 Boudreault, F., Pinilla-Vera, M., Englert, J.A., Kho, A.T., Isabelle, C., Arciniegas, A.J., Barragan-Bradford, D., Quintana, C., Amador-Munoz, D., Guan, J., Choi, K.M., Registry, M., Sholl, L., Hurwitz, S., Tschumperlin, D.J., and Baron, R.M. (2017). Zinc deficiency primes the lung for ventilator-induced injury. JCI Insight 2.

Bueno, M., Brands, J., Voltz, L., Fiedler, K., Mays, B., St Croix, C., Sembrat, J., Mallampalli, R.K., Rojas, M., and Mora, A.L. (2018). ATF3 represses PINK1 gene transcription in lung epithelial cells to control mitochondrial homeostasis. Aging Cell 17.

Bueno, M., Lai, Y.C., Romero, Y., Brands, J., St Croix, C.M., Kamga, C., Corey, C., Herazo-Maya, J.D., Sembrat, J., Lee, J.S., Duncan, S.R., Rojas, M., Shiva, S., Chu, C.T., and Mora, A.L. (2015). PINK1 deficiency impairs mitochondrial homeostasis and promotes lung fibrosis. J Clin Invest 125, 521-538.

Cander, B., Dundar, Z.D., Gul, M., and Girisgin, S. (2011). Prognostic value of serum zinc levels in critically ill patients. J Crit Care 26, 42-46.

Chen, H., Matsumoto, K., Brockway, B.L., Rackley, C.R., Liang, J., Lee, J.H., Jiang, D., Noble, P.W., Randell, S.H., Kim, C.F., and Stripp, B.R. (2012a). Airway epithelial progenitors are region specific and show differential responses to bleomycininduced lung injury. Stem Cells 30, 1948-1960.

Chen, X., Bian, J., and Ge, Y. (2012b). Zinc-deficient diet aggravates ventilation-induced lung injury in rats. J Biomed Res 26, 59-65.

840 Desai, T.J., Brownfield, D.G., and Krasnow, M.A. (2014). Alveolar progenitor and stem cells in lung development, renewal and cancer. Nature 507, 190-194. 
Fagerberg, L., Hallstrom, B.M., Oksvold, P., Kampf, C., Djureinovic, D., Odeberg, J., Habuka, M., Tahmasebpoor, S., Danielsson, A., Edlund, K., Asplund, A., Sjostedt, E., Lundberg, E., Szigyarto, C.A., Skogs, M., Takanen, J.O., Berling, H., Tegel, H., Mulder, J., Nilsson, P., Schwenk, J.M., Lindskog, C., Danielsson, F., Mardinoglu, A., Sivertsson, A., von Feilitzen, K., Forsberg, M., Zwahlen, M., Olsson, I., Navani, S., Huss, M., Nielsen, J., Ponten, F., and Uhlen, M. (2014). Analysis of the human tissue-specific expression by genome-wide integration of transcriptomics and antibody-based proteomics. Mol Cell Proteomics 13, 397-406.

Geng, Y., Liu, X., Liang, J., Habiel, D.M., Kulur, V., Coelho, A.L., Deng, N., Xie, T., Wang, Y., Liu, N., Huang, G., Kurkciyan, A., Liu, Z., Tang, J., Hogaboam, C.M., Jiang, D., and Noble, P.W. (2019). PD-L1 on invasive fibroblasts drives fibrosis in a humanized model of idiopathic pulmonary fibrosis. JCI Insight 4.

Gonzalez, R.F., Allen, L., Gonzales, L., Ballard, P.L., and Dobbs, L.G. (2010). HTII-280, a biomarker specific to the apical plasma membrane of human lung alveolar type II cells. J Histochem Cytochem 58, 891-901.

Habermann, A.C., Gutierrez, A.J., Bui, L.T., Yahn, S.L., Winters, N.I., Calvi, C.L., Peter, L., Chung, M.-I., Taylor, C.J., Jetter, C., Raju, L., Roberson, J., Ding, G., Wood, L., Sucre, J.M.S., Richmond, B.W., Serezani, A.P., McDonnell, W.J., Mallal, S.B., Bacchetta, M.J., Loyd, J.E., Shaver, C.M., Ware, L.B., Bremner, R., Walia, R., Blackwell, T.S., Banovich, N.E., and Kropski, J.A. (2020). Single-cell RNA sequencing reveals profibrotic roles of distinct epithelial and mesenchymal lineages in pulmonary fibrosis. Science Advances 6, eaba1972.

Hershberger, K.A., Martin, A.S., and Hirschey, M.D. (2017). Role of NAD(+) and mitochondrial sirtuins in cardiac and renal diseases. Nat Rev Nephrol 13, 213-225.

Hogan, B.L., Barkauskas, C.E., Chapman, H.A., Epstein, J.A., Jain, R., Hsia, C.C., Niklason, L., Calle, E., Le, A., Randell, S.H., Rock, J., Snitow, M., Krummel, M., Stripp, B.R., Vu, T., White, E.S., Whitsett, J.A., and Morrisey, E.E. (2014). Repair and regeneration of the respiratory system: complexity, plasticity, and mechanisms of lung stem cell function. Cell Stem Cell 15, 123-138.

Imai, S., and Guarente, L. (2014). NAD+ and sirtuins in aging and disease. Trends Cell Biol 24, 464-471.

Jiang, D., Liang, J., Hodge, J., Lu, B., Zhu, Z., Yu, S., Fan, J., Gao, Y., Yin, Z., Homer, R., Gerard, C., and Noble, P.W. (2004). Regulation of pulmonary fibrosis by chemokine receptor CXCR3. The Journal of clinical investigation 114, 291-299.

Jiang, D., Liang, J., and Noble, P.W. (2020). Stem Cells and Progenitor Cells in Interstitial Lung Disease. In Encyclopedia of Respiratory Medicine, 2nd Edition (Elsevier).

Jiang, L.J., Maret, W., and Vallee, B.L. (1998). The glutathione redox couple modulates zinc transfer from metallothionein to zinc-depleted sorbitol dehydrogenase. Proc Natl Acad Sci U S A 95, 3483-3488.

Kambe, T., Tsuji, T., Hashimoto, A., and Itsumura, N. (2015). The Physiological, Biochemical, and Molecular Roles of Zinc Transporters in Zinc Homeostasis and Metabolism. Physiol Rev 95, 749-784.

Knoell, D.L., Julian, M.W., Bao, S., Besecker, B., Macre, J.E., Leikauf, G.D., DiSilvestro, R.A., and Crouser, E.D. (2009). Zinc deficiency increases organ damage and mortality in a murine model of polymicrobial sepsis. Crit Care Med 37, 1380-1388. 
Korfei, M., Ruppert, C., Mahavadi, P., Henneke, I., Markart, P., Koch, M., Lang, G., Fink, L., Bohle, R.M., Seeger, W., Weaver, T.E., and Guenther, A. (2008). Epithelial endoplasmic reticulum stress and apoptosis in sporadic idiopathic pulmonary fibrosis. Am J Respir Crit Care Med 178, 838-846.

Kramer, A., Green, J., Pollard, J., Jr., and Tugendreich, S. (2014). Causal analysis approaches in Ingenuity Pathway Analysis. Bioinformatics 30, 523-530.

Larson-Casey, J.L., Deshane, J.S., Ryan, A.J., Thannickal, V.J., and Carter, A.B. (2016). Macrophage Akt1 Kinase-Mediated Mitophagy Modulates Apoptosis Resistance and Pulmonary Fibrosis. Immunity 44, 582-596.

Lawson, W.E., Cheng, D.S., Degryse, A.L., Tanjore, H., Polosukhin, V.V., Xu, X.C., Newcomb, D.C., Jones, B.R., Roldan, J., Lane, K.B., Morrisey, E.E., Beers, M.F., Yull, F.E., and Blackwell, T.S. (2011). Endoplasmic reticulum stress enhances fibrotic remodeling in the lungs. Proc Natl Acad Sci U S A 108, 10562-10567.

Lee, H.Y., and Hong, I.S. (2020). Metabolic regulation and its molecular mechanisms in various stem cell functions. Curr Stem Cell Res Ther.

Leibiger, I.B., and Berggren, P.O. (2006). Sirt1: a metabolic master switch that modulates lifespan. Nat Med 12, 34-36; discussion 36.

Liang, J., Liu, N., Liu, X., Mena, J.M., Xie, T., Geng, Y., Huan, C., Zhang, Y., Taghavifar, F., Huang, G., Kurkciyan, A., Barron, V., Jiang, D., and Noble, P.W. (2019). Mitogen-activated Protein Kinase-activated Protein Kinase 2 Inhibition Attenuates Fibroblast Invasion and Severe Lung Fibrosis. Am J Respir Cell Mol Biol 60, 4148.

Liang, J., Zhang, Y., Xie, T., Liu, N., Chen, H., Geng, Y., Kurkciyan, A., Mena, J.M., Stripp, B.R., Jiang, D., and Noble, P.W. (2016). Hyaluronan and TLR4 promote surfactantprotein-C-positive alveolar progenitor cell renewal and prevent severe pulmonary fibrosis in mice. Nat Med 22, 1285-1293.

Liu, M.J., Bao, S., Galvez-Peralta, M., Pyle, C.J., Rudawsky, A.C., Pavlovicz, R.E., Killilea, D.W., Li, C., Nebert, D.W., Wewers, M.D., and Knoell, D.L. (2013). ZIP8 regulates host defense through zinc-mediated inhibition of NF-kappaB. Cell Rep 3, 386-400.

Martinez, F.J., Collard, H.R., Pardo, A., Raghu, G., Richeldi, L., Selman, M., Swigris, J.J., Taniguchi, H., and Wells, A.U. (2017). Idiopathic pulmonary fibrosis. Nat Rev Dis Primers 3, 17074.

Minagawa, S., Araya, J., Numata, T., Nojiri, S., Hara, H., Yumino, Y., Kawaishi, M., Odaka, M., Morikawa, T., Nishimura, S.L., Nakayama, K., and Kuwano, K. (2011). Accelerated epithelial cell senescence in IPF and the inhibitory role of SIRT6 in TGF-beta-induced senescence of human bronchial epithelial cells. Am J Physiol Lung Cell Mol Physiol 300, L391-401.

Naikawadi, R.P., Disayabutr, S., Mallavia, B., Donne, M.L., Green, G., La, J.L., Rock, J.R., Looney, M.R., and Wolters, P.J. (2016). Telomere dysfunction in alveolar epithelial cells causes lung remodeling and fibrosis. JCI Insight 1, e86704.

Noble, P.W., Barkauskas, C.E., and Jiang, D. (2012). Pulmonary fibrosis: patterns and perpetrators. The Journal of clinical investigation 122, 2756-2762.

Nogueiras, R., Habegger, K.M., Chaudhary, N., Finan, B., Banks, A.S., Dietrich, M.O., Horvath, T.L., Sinclair, D.A., Pfluger, P.T., and Tschop, M.H. (2012). Sirtuin 1 and sirtuin 3: physiological modulators of metabolism. Physiol Rev 92, 1479-1514. 
O'Callaghan, C., and Vassilopoulos, A. (2017). Sirtuins at the crossroads of stemness, aging, and cancer. Aging Cell 16, 1208-1218.

Ohashi, W., Hara, T., Takagishi, T., Hase, K., and Fukada, T. (2019). Maintenance of Intestinal Epithelial Homeostasis by Zinc Transporters. Dig Dis Sci 64, 2404-2415.

Ohashi, W., Kimura, S., Iwanaga, T., Furusawa, Y., Irie, T., Izumi, H., Watanabe, T., Hijikata, A., Hara, T., Ohara, O., Koseki, H., Sato, T., Robine, S., Mori, H., Hattori, Y., Watarai, H., Mishima, K., Ohno, H., Hase, K., and Fukada, T. (2016). Zinc Transporter SLC39A7/ZIP7 Promotes Intestinal Epithelial Self-Renewal by Resolving ER Stress. PLoS Genet 12, e1006349.

Prasad, A.S. (2008). Zinc in human health: effect of zinc on immune cells. Mol Med 14, 353-357.

Prasad, A.S., Fitzgerald, J.T., Hess, J.W., Kaplan, J., Pelen, F., and Dardenne, M. (1993). Zinc deficiency in elderly patients. Nutrition 9, 218-224.

Price, N.L., Gomes, A.P., Ling, A.J., Duarte, F.V., Martin-Montalvo, A., North, B.J., Agarwal, B., Ye, L., Ramadori, G., Teodoro, J.S., Hubbard, B.P., Varela, A.T., Davis, J.G., Varamini, B., Hafner, A., Moaddel, R., Rolo, A.P., Coppari, R., Palmeira, C.M., de Cabo, R., Baur, J.A., and Sinclair, D.A. (2012). SIRT1 is required for AMPK activation and the beneficial effects of resveratrol on mitochondrial function. Cell Metab 15, 675-690.

Raghu, G., Chen, S.Y., Hou, Q., Yeh, W.S., and Collard, H.R. (2016). Incidence and prevalence of idiopathic pulmonary fibrosis in US adults 18-64 years old. Eur Respir J 48, 179-186.

Ren, R., Ocampo, A., Liu, G.H., and Izpisua Belmonte, J.C. (2017). Regulation of Stem Cell Aging by Metabolism and Epigenetics. Cell Metab 26, 460-474.

Rojas, M., Mora, A.L., Kapetanaki, M., Weathington, N., Gladwin, M., and Eickelberg, O. (2015). Aging and Lung Disease. Clinical Impact and Cellular and Molecular Pathways. Ann Am Thorac Soc 12, S222-227.

Ryu, C., Sun, H., Gulati, M., Herazo-Maya, J.D., Chen, Y., Osafo-Addo, A., Brandsdorfer, C., Winkler, J., Blaul, C., Faunce, J., Pan, H., Woolard, T., Tzouvelekis, A., AntinOzerkis, D.E., Puchalski, J.T., Slade, M., Gonzalez, A.L., Bogenhagen, D.F., Kirillov, V., Feghali-Bostwick, C., Gibson, K., Lindell, K., Herzog, R.I., Dela Cruz, C.S., Mehal, W., Kaminski, N., Herzog, E.L., and Trujillo, G. (2017). Extracellular Mitochondrial DNA Is Generated by Fibroblasts and Predicts Death in Idiopathic Pulmonary Fibrosis. Am J Respir Crit Care Med 196, 1571-1581.

Selman, M., Buendia-Roldan, I., and Pardo, A. (2016). Aging and Pulmonary Fibrosis. Rev Invest Clin 68, 75-83.

Selman, M., and Pardo, A. (2014). Revealing the pathogenic and aging-related mechanisms of the enigmatic idiopathic pulmonary fibrosis. an integral model. Am J Respir Crit Care Med 189, 1161-1172.

Seto, E., and Yoshida, M. (2014). Erasers of histone acetylation: the histone deacetylase enzymes. Cold Spring Harb Perspect Biol 6, a018713.

Shyh-Chang, N., and Ng, H.H. (2017). The metabolic programming of stem cells. Genes Dev 31, 336-346.

975 Sosulski, M.L., Gongora, R., Danchuk, S., Dong, C., Luo, F., and Sanchez, C.G. (2015). Deregulation of selective autophagy during aging and pulmonary fibrosis: the role of TGFbeta1. Aging Cell 14, 774-783. 
Tan, B.L., Norhaizan, M.E., Liew, W.P., and Sulaiman Rahman, H. (2018). Antioxidant and Oxidative Stress: A Mutual Interplay in Age-Related Diseases. Front Pharmacol 9, 1162.

Tevy, M.F., Giebultowicz, J., Pincus, Z., Mazzoccoli, G., and Vinciguerra, M. (2013). Aging signaling pathways and circadian clock-dependent metabolic derangements. Trends Endocrinol Metab 24, 229-237.

Thannickal, V.J. (2013). Mechanistic links between aging and lung fibrosis. Biogerontology 14, 609-615.

Tian, Y., Li, H., Qiu, T., Dai, J., Zhang, Y., Chen, J., and Cai, H. (2019). Loss of PTEN induces lung fibrosis via alveolar epithelial cell senescence depending on NFkappaB activation. Aging Cell 18, e12858.

Vaquero, A., Scher, M., Lee, D., Erdjument-Bromage, H., Tempst, P., and Reinberg, D. (2004). Human SirT1 interacts with histone $\mathrm{H} 1$ and promotes formation of facultative heterochromatin. Mol Cell 16, 93-105.

Vasto, S., Mocchegiani, E., Malavolta, M., Cuppari, I., Listi, F., Nuzzo, D., Ditta, V., Candore, G., and Caruso, C. (2007). Zinc and inflammatory/immune response in aging. Ann N Y Acad Sci 1100, 111-122.

Xie, T., Wang, Y., Deng, N., Huang, G., Taghavifar, F., Geng, Y., Liu, N., Kulur, V., Yao, C., Chen, P., Liu, Z., Stripp, B., Tang, J., Liang, J., Noble, P.W., and Jiang, D. (2018). Single-Cell Deconvolution of Fibroblast Heterogeneity in Mouse Pulmonary Fibrosis. Cell Rep 22, 3625-3640.

Xu, Y., Mizuno, T., Sridharan, A., Du, Y., Guo, M., Tang, J., Wikenheiser-Brokamp, K.A., Perl, A.T., Funari, V.A., Gokey, J.J., Stripp, B.R., and Whitsett, J.A. (2016). Singlecell RNA sequencing identifies diverse roles of epithelial cells in idiopathic pulmonary fibrosis. JCI Insight 1, e90558.

Yaku, K., Okabe, K., and Nakagawa, T. (2018). NAD metabolism: Implications in aging and longevity. Ageing Res Rev 47, 1-17.

1005 Yang, M., Bao, D., Shi, A., Yuan, H., Wang, J., He, W., Tong, X., and Qin, H. (2020). Zinc promotes patient-derived induced pluripotent stem cell neural differentiation via ERK-STAT signaling. Stem Cells Dev.

Zhang, J., Zhao, J., Dahan, P., Lu, V., Zhang, C., Li, H., and Teitell, M.A. (2018). Metabolism in Pluripotent Stem Cells and Early Mammalian Development. Cell Metab 27, 332-338. 
Figure 1

A

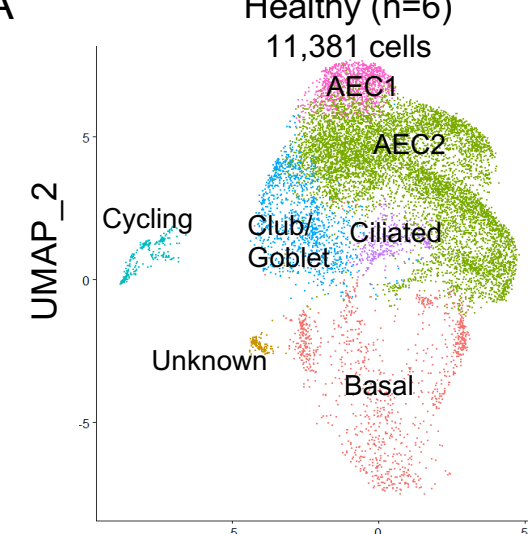

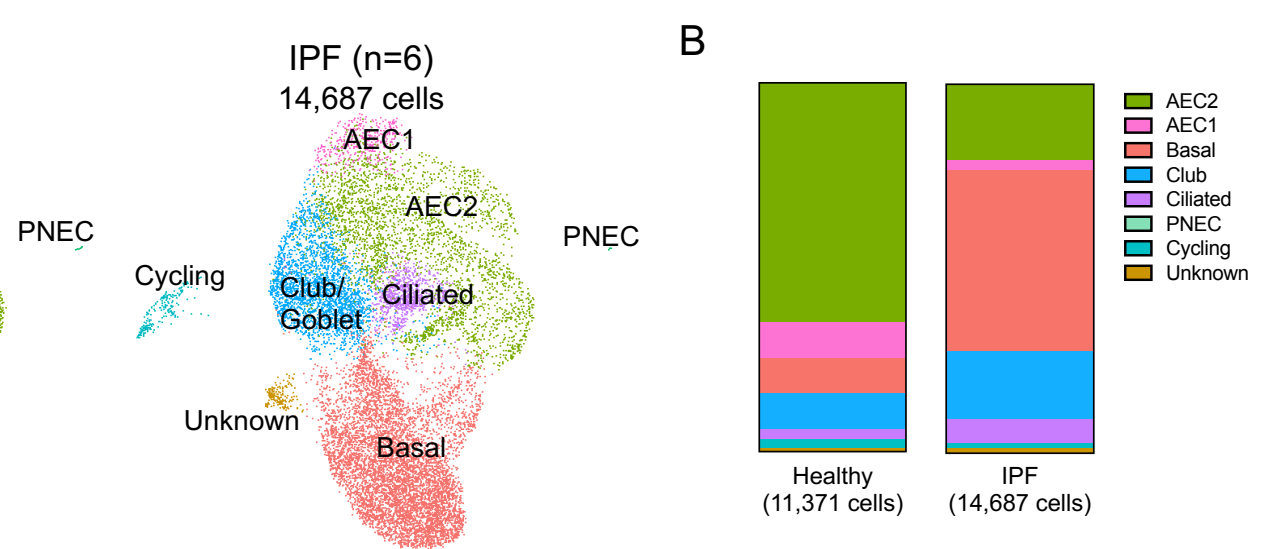

C

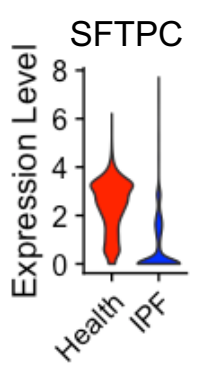

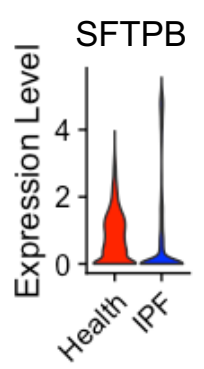

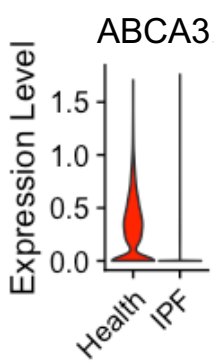

$\mathrm{D}$

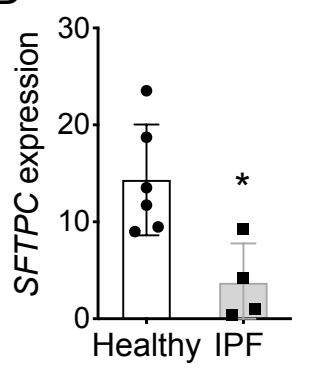

G

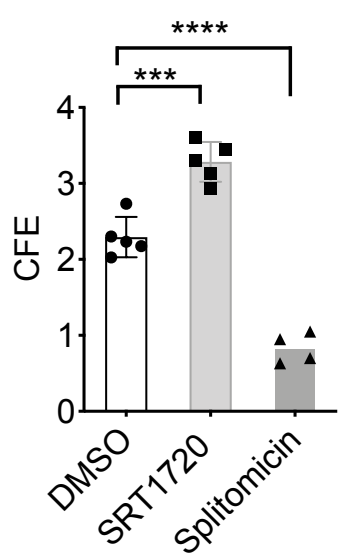

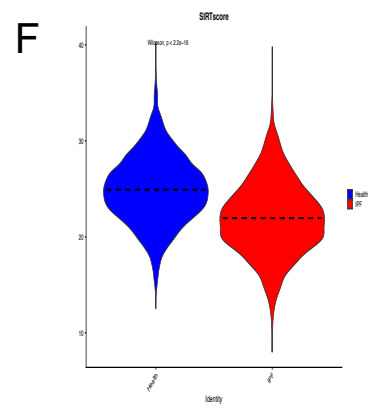

$\mathrm{E}$

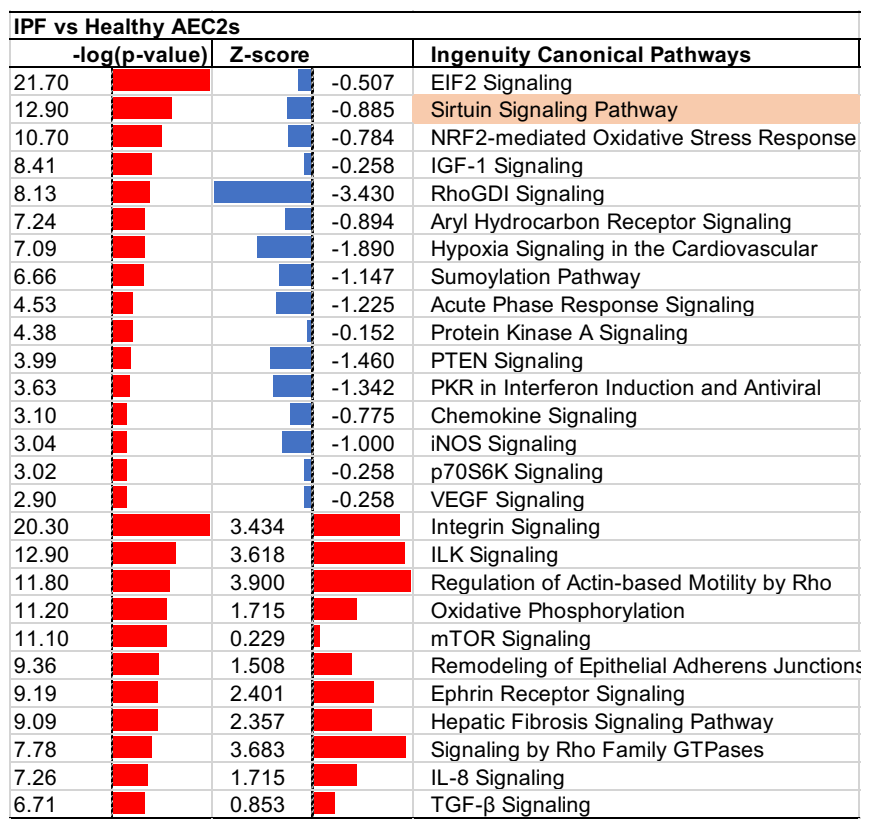

$\mathrm{H}$
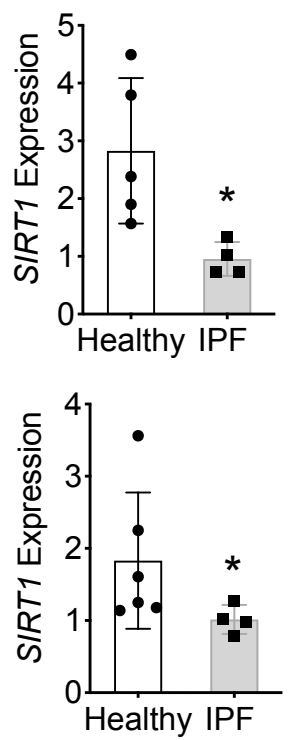

Figure 1. scRNA-seq identifies dysregulation of metabolism and stem cell signaling in IPF AEC2s (A) UMAP plots of flow enriched EpCAM+CD31-CD45- cells from healthy $(11,381$ cells, $n=6)$ and IPF lungs (14,687 cells, $n=6$ ). (B) Distribution of epithelial cell types. (C) Expression of AEC2 marker genes from healthy and IPF lungs. (D) SFTPC expression in healthy and IPF AEC2s by RT-PCR ( $n=6$ and $\left.4,{ }^{*} p<0.05\right)$. (E) IPA pathway analysis of AEC2s from healthy and IPF lungs. (F) Sirtuin activation score of healthy and IPF AEC2s. (G) 3D organoid culture of AEC2s from healthy lungs treated with SRT1720 and splitomicin ( $n=4-$ 5 , $\left.{ }^{* * *} \mathrm{p}<0.001,{ }^{* * * *} \mathrm{p}<0.0001\right)$. (H,I) SIRT1 expression in fresh isolated AEC2s $(\mathrm{H})$ and AEC2s derived from 3 D organoids (I) by qPCR $\left(n=4-6,{ }^{*} p<0.05\right)$. 


\section{Figure 2}

A

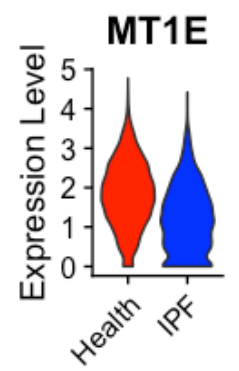

$E$

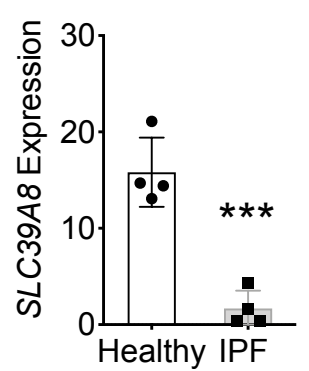

$\mathrm{H}$

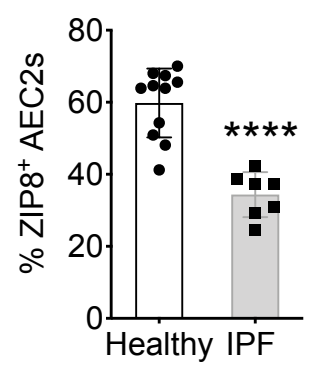

$\mathrm{F}$

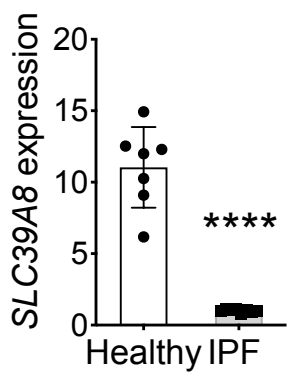

I
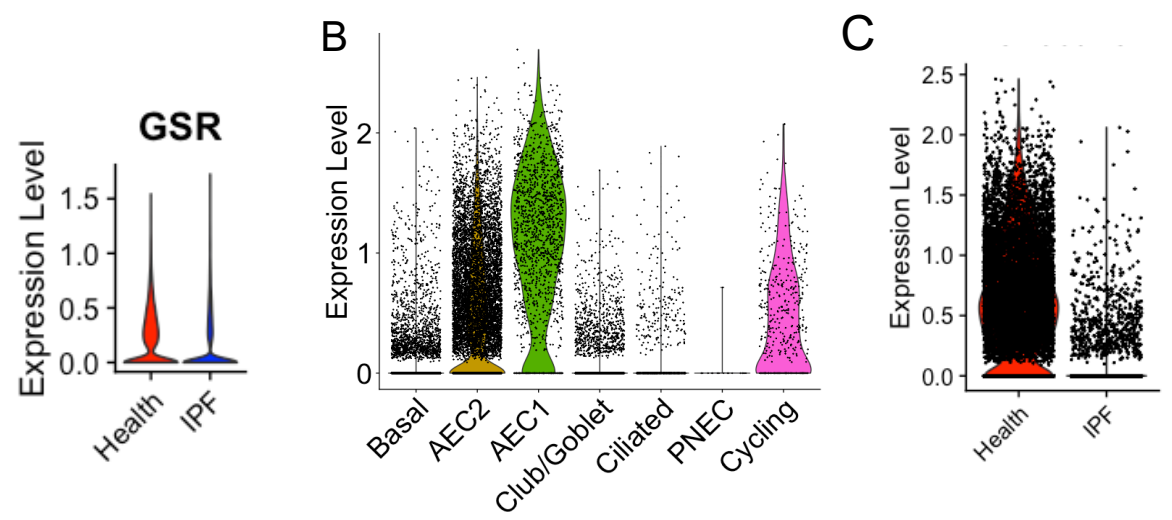

G

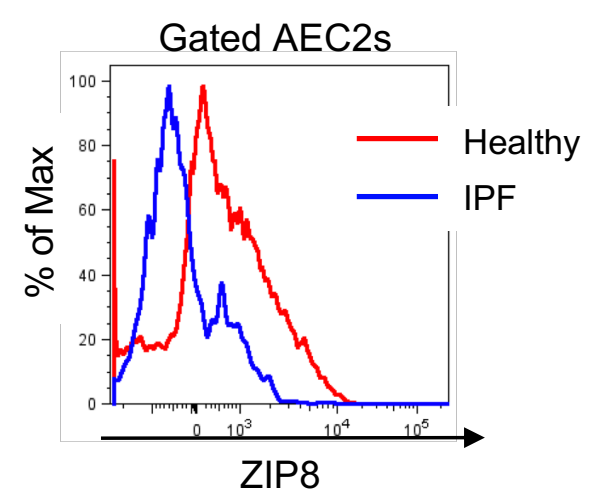

$J$

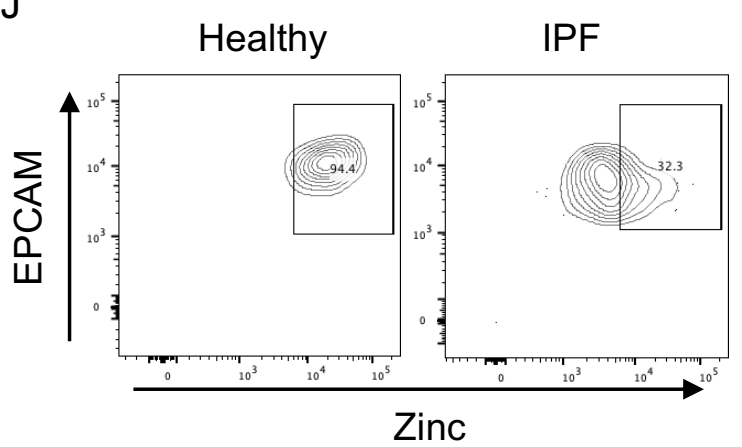

Figure 2. Dysregulation of zinc metabolism of IPF AEC2s.

(A) scRNA-seq own regulated zinc metabolism related genes in IPF AEC2s.

(B) SLC39A8 expression of human lung epithelial cell types.

(C) SLC39A8 expression of AEC2s from healthy and IPF lungs.

(E) SLC39A8 expression of AEC2s freshly isolated from lung tissues ( $n=4$ each. $\left.{ }^{* * *} p<0.001\right)$.

(E) SLC39A8 expression of AEC2s derived from 3D cultured organoids ( $\left.n=7-8 .{ }^{* * * *} p<0.0001\right)$.

$(F, G)$ Flow cytometry of cell surface ZIP8 level and percent of ZIP8+ cells of healthy and IPF AEC2s.

( $\left.\mathrm{n}=7-11,{ }^{* * * *} \mathrm{p}<0.0001\right)$.

(H) SLC39A8 expression of healthy and ILD lung tissues $\left(n=137-255\right.$, $\left.{ }^{* \star * *} p<0.0001\right)$.

(I) Representative plots of intracellular zinc staining of human AEC2s. 


\section{Figure 3}

A

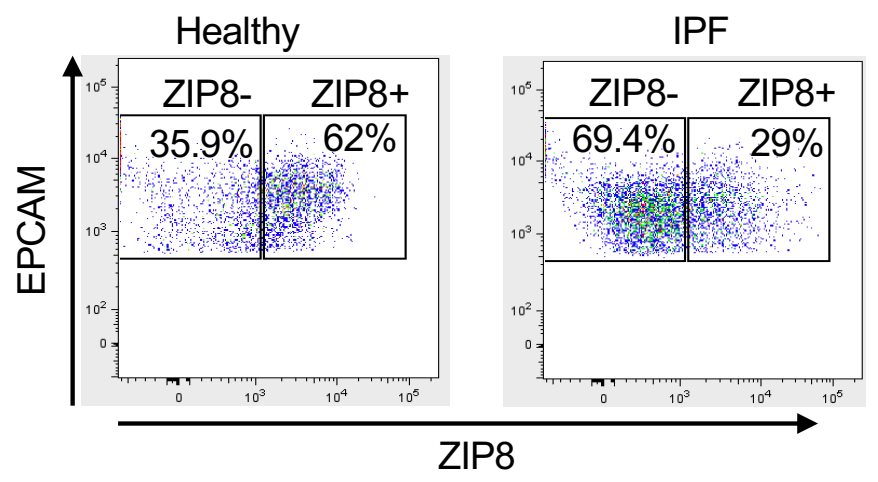

B

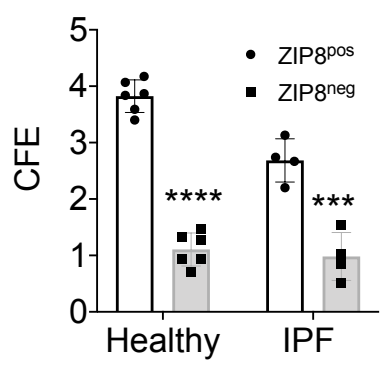

C

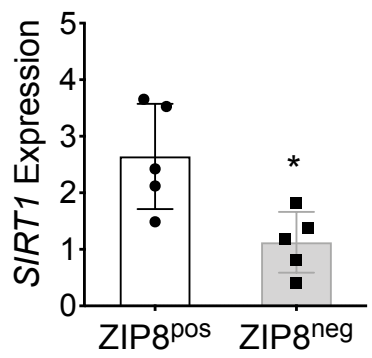

D

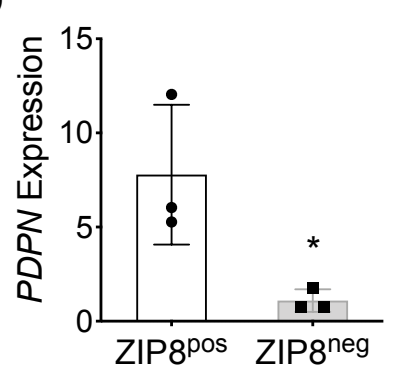

$\mathrm{H}$

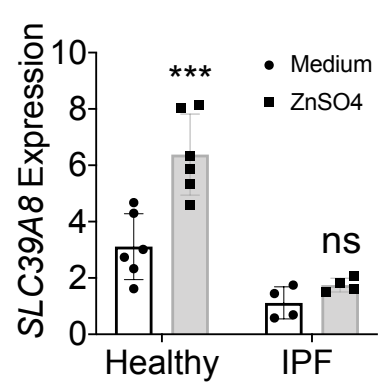

$\mathrm{E}$

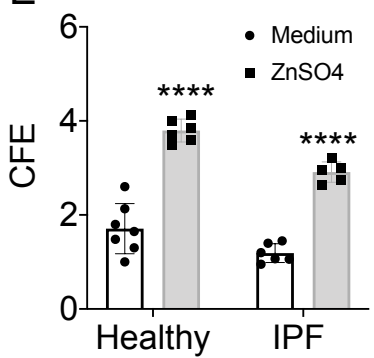

I

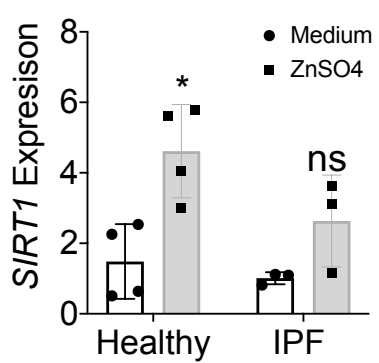

$\mathrm{F}$

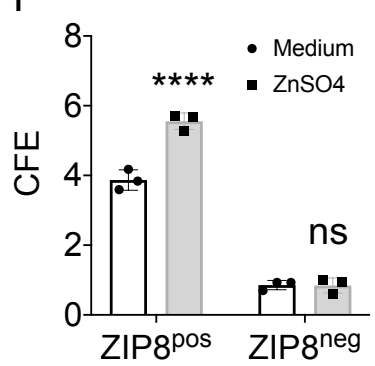

G

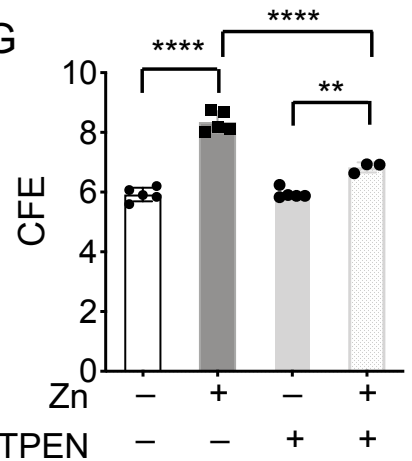

$J$

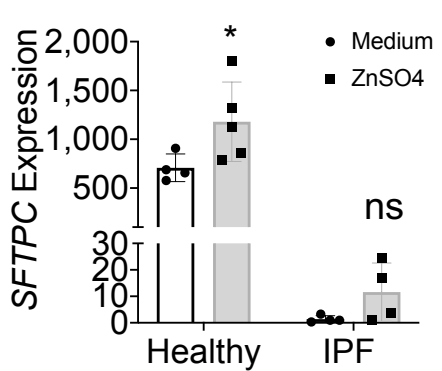

Figure 3. ZIP8 expression is associated with AEC2 renewal.

A) Flow cytometry plots of gated ZIP8+ and ZIP8- AEC2s.

(B) CFE of flow sorted ZIP8+ and ZIP8- AEC2s from healthy and IPF lungs $\left(n=4-6,{ }^{* * *} p<0.001,{ }^{* * * *} p\right.$ $<0.0001)$.

$(\mathrm{C}, \mathrm{D}) \operatorname{SIRT1}\left(\mathrm{C}, \mathrm{n}=5\right.$ each, $\left.{ }^{*} \mathrm{p}<0.05\right)$.) and PDPN (D, $\mathrm{n}=3$ each, * $\left.\mathrm{p}<0.05\right)$.) expression of AEC2s derived from $3 D$ cultured organoids of ZIP8+ and ZIP8- AEC2s from healthy lung.

(E) CFE of AEC2s from healthy and IPF lungs with and without zinc sulfate $(100 \mathrm{mM})$ treatment $(n=5-$ $\left.7,{ }^{* * * *} p<0.0001\right)$.

(F) CFE of ZIP8 ${ }^{+}$and ZIP8- AEC2s with and without zinc sulfate $(100 \mathrm{mM})$ treatment $\left(\mathrm{n}=3\right.$ each. ${ }^{* * *} \mathrm{p}<$ 0.0001 ; ns, not significant).

(G) CFE of AEC2s with and without zinc sulfate and TPEN treatment $\left(n=3-5,{ }^{* *} p<0.01,{ }^{* * *} p<\right.$ $0.0001)$.

(H-J) Expression of SLC39A8 $\left(n=4-6,{ }^{* * *} p<0.001\right)$, SIRT1 $\left(n=3-4,{ }^{*} p<0.05\right)$ and SFTPC $(n=4-$ $\left.5,{ }^{*} p<0.05\right)$ in AEC2s with and without zinc sulfate by RT-PCR (ns, not significant). 


\section{Figure 4}

A
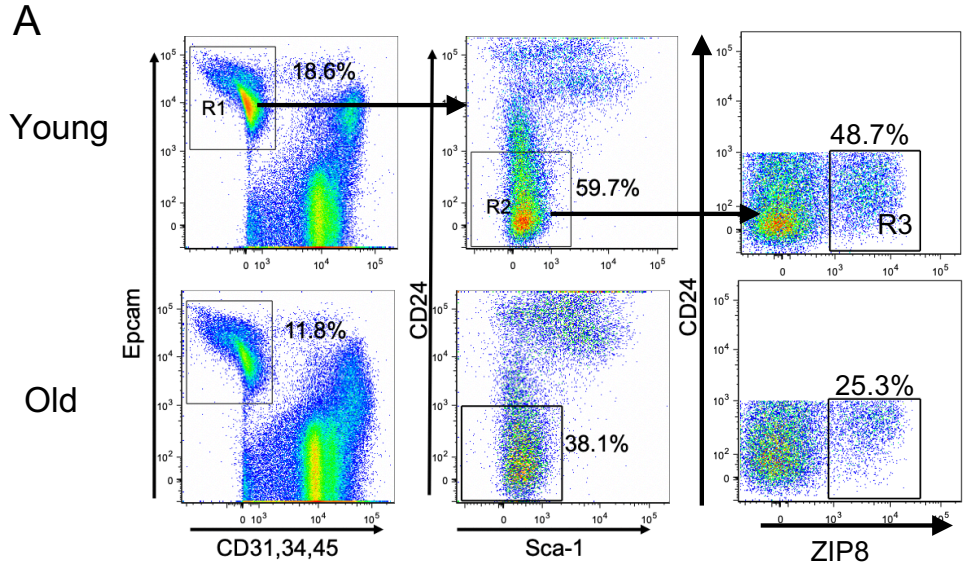

B
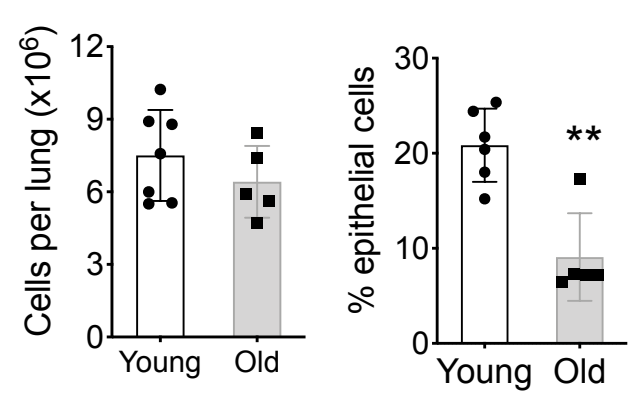

$\mathrm{D}$

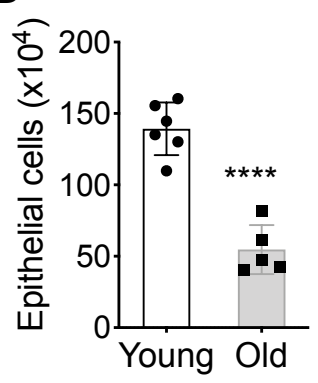

$E$

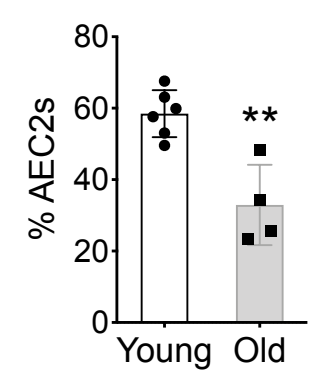

$\mathrm{F}$

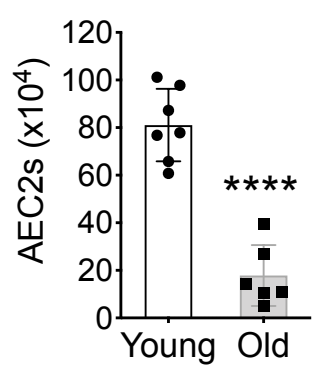

G

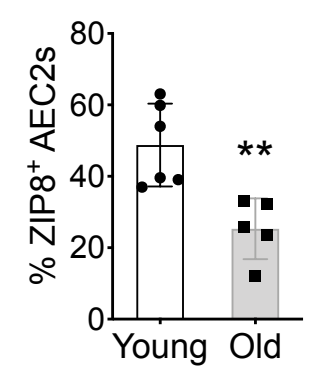

$\mathrm{H}$

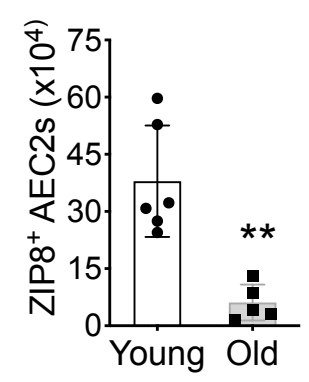

I

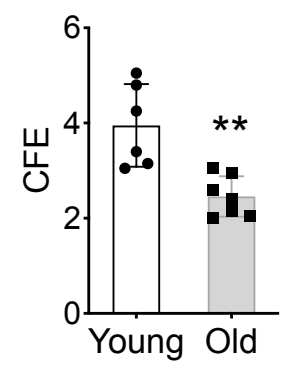

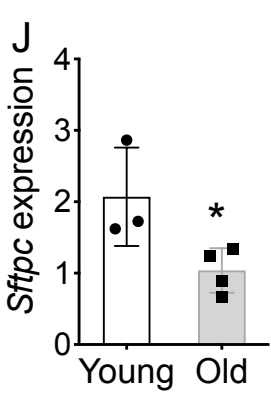

K

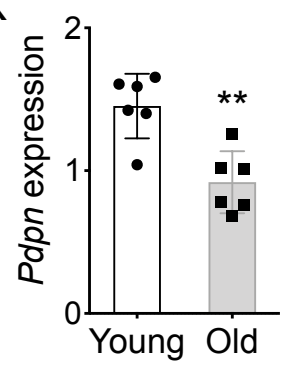

Figure 4. Decreased ZIP8 expression and renewal capacity of aging AEC2s.

(A) Gating strategy for ZIP8 expressing AEC2s from young and old mice by flow cytometry.

(B) Number of total cells recovered from young and old mouse lungs ( $n=5-6$ ).

(C, D) Percent and number of EpCAM+CD31-CD34-CD45- epithelial cells (R1 in panel $A)\left(n=5-6\right.$. ${ }^{* *} p<$ $\left.0.01,{ }^{* * * *} p<0.0001\right)$.

(E) Percent of CD24-Sca-1- AEC2s (R2 in panel A) within total lung epithelial cells (R1 in panel $A)(n=$ $\left.4-6,{ }^{* *} p<0.01\right)$.

(F) Number of AEC2s were recovered from young and old mouse lung ( $\left.n=6-7,{ }^{* * * *} p<0.0001\right)$.

(G) Percent of ZIP8+ cells within total AEC2s (R3 in panel A) $\left(n=5-6,{ }^{* *} p<0.01\right)$.

(H) Number of ZIP8+ cells were recovered from young and old mouse lung $\left(n=5-6,{ }^{* *} p<0.01\right)$.

(I) CEF of mouse AEC2s isolated from young and old mouse lungs ( $n=6-7,{ }^{* *} p<0.01$ ).

$(\mathrm{J}, \mathrm{K})$ Expression of Sftpc $(\mathrm{J})\left(\mathrm{n}=3-4,{ }^{*} \mathrm{p}<0.05\right)$ and Pdpn $(\mathrm{K})\left(\mathrm{n}=6\right.$ each, $\left.{ }^{* *} \mathrm{p}<0.01\right)$ of mouse AEC2s derived from 3D cultured organoids by RT-PCR. 
Fig 5

A

\begin{tabular}{|c|c|c|}
\hline \multicolumn{3}{|c|}{ Old Day4 vs. Young Day4 } \\
\hline$-\log (p-v a l u e)$ & z-score & Ingenuity Canonical Pathways \\
\hline 32.00 & -0.566 & EIF2 Signaling \\
\hline 18.60 & -2.940 & Sirtuin Signaling Pathway \\
\hline 11.90 & -1.225 & mTOR Signaling \\
\hline 11.50 & -2.117 & NRF2-mediated Oxidative Stress Response \\
\hline 6.56 & -1.897 & Hypoxia Signaling in the Cardio. System \\
\hline 4.80 & -2.646 & PI3K/AKT Signaling \\
\hline 3.28 & -1.291 & Cdc42 Signaling \\
\hline 3.18 & -2.263 & Integrin Signaling \\
\hline 3.15 & -1.698 & p53 Signaling \\
\hline 3.00 & -2.828 & Glycolysis I \\
\hline 2.68 & -1.667 & Cell Cycle: G2/M DNA Damage Checkpoint \\
\hline 2.40 & -1.414 & Signaling by Rho Family GTPases \\
\hline 1.89 & -1.213 & Apoptosis Signaling \\
\hline 29.90 & & Mitochondrial Dysfunction \\
\hline 9.38 & & Protein Ubiquitination Pathway \\
\hline 5.63 & & Unfolded protein response \\
\hline 3.83 & & Epithelial Adherens Junction Signaling \\
\hline 28.40 & 5.171 & Oxidative Phosphorylation \\
\hline 7.39 & 2.887 & Glutathione Redox Reactions I \\
\hline 4.22 & 3.162 & Glutathione-mediated Detoxification \\
\hline 3.15 & 2.668 & Apelin Adipocyte Signaling Pathway \\
\hline 3.09 & 2.357 & LXR/RXR Activation \\
\hline 1.89 & 2.111 & Lymphotoxin $\beta$ Receptor Signaling \\
\hline 1.77 & 2.000 & Oleate Biosynthesis II (Animals) \\
\hline 1.72 & 2.357 & iCOS-iCOSL Signaling in T Helper Cells \\
\hline 1.51 & 1.890 & Fatty Acid $\beta$-oxidation I \\
\hline
\end{tabular}

B

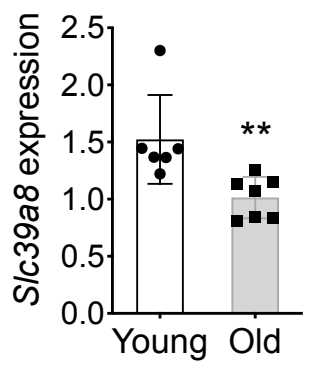

D

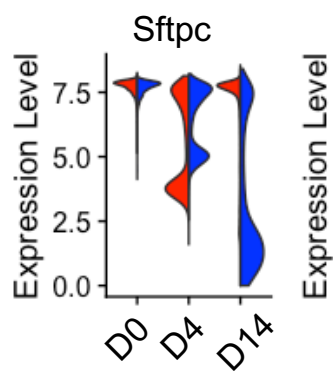

C

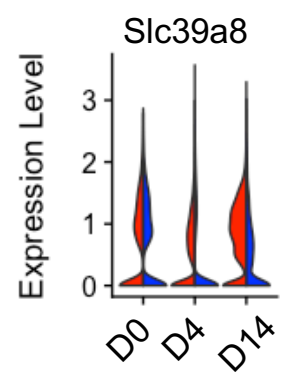

$\mathrm{E}$

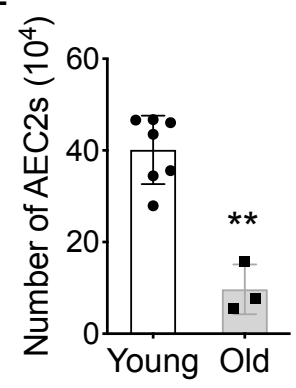

Figure 5. Decreased ZIP8 expression and renewal capacity of aging AEC2s.

(A) IPA pathway analysis of AEC2s from young and aged mice 4 days after bleomycin.

(B) qPCR S/c39a8 expression of AEC2s from uninjured mouse lungs ( $n=6-7,{ }^{* *} p<0.01$ ).

(C, D) Expression of S/c39a8 (C) and AEC2 marker genes Sftpc and Abca3 (D) of AEC2s from day 0 and bleomycin day 4 , day 14 young and old mouse lungs.

(E) Number of AEC2s were recovered from mouse lung tissue at day 14 after bleomycin injury $(n=3-$ $\left.6,{ }^{* * *} p<0.001\right)$. 
Figure 6

A

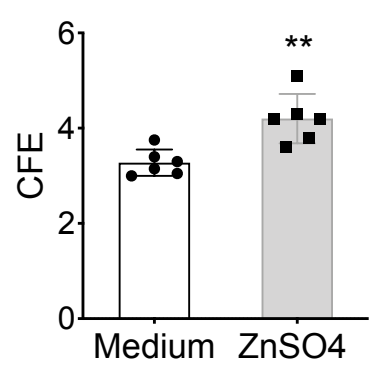

D

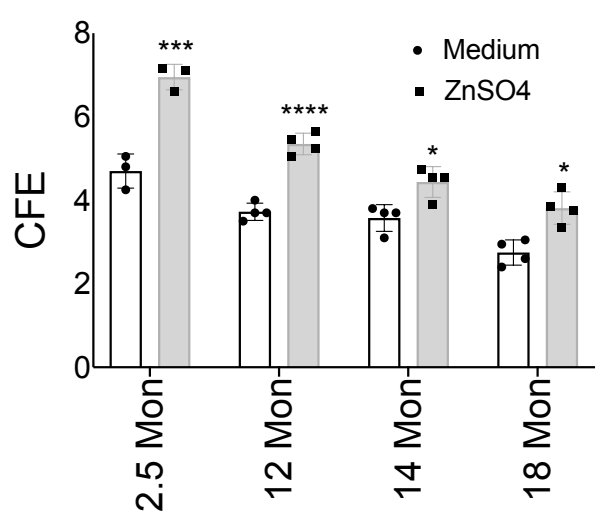

$\mathrm{F}$

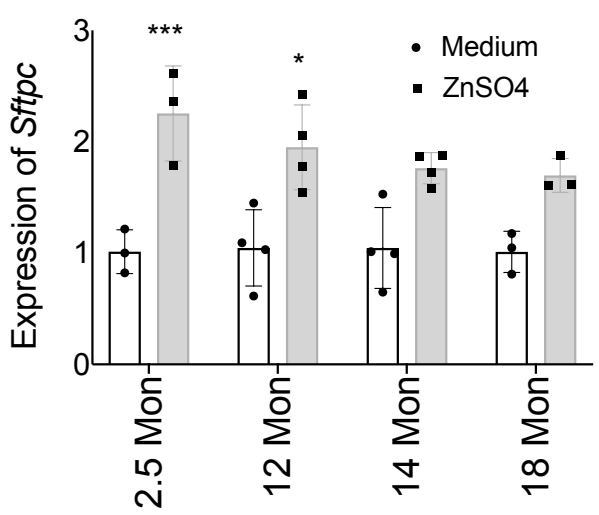

$\mathrm{B}$

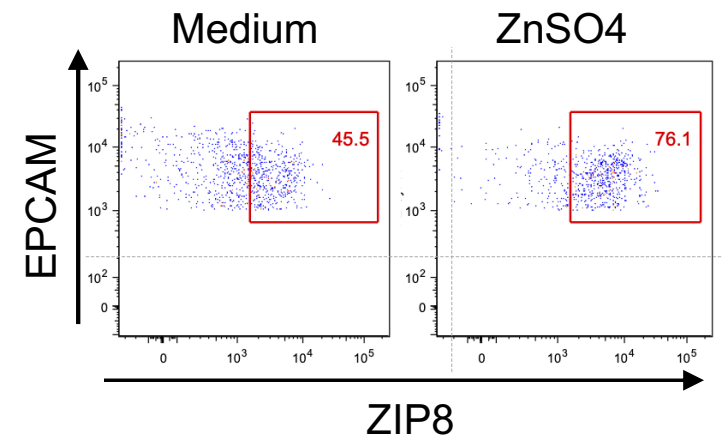

C

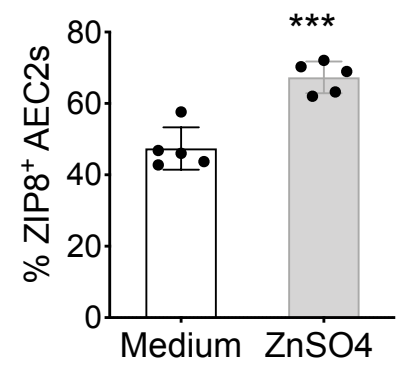

$E$

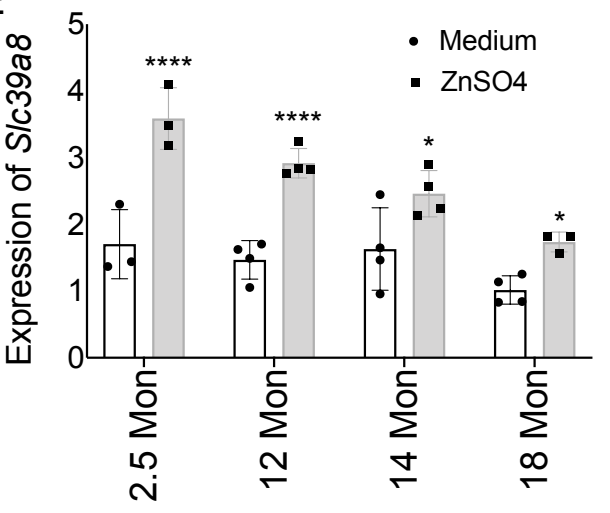

G

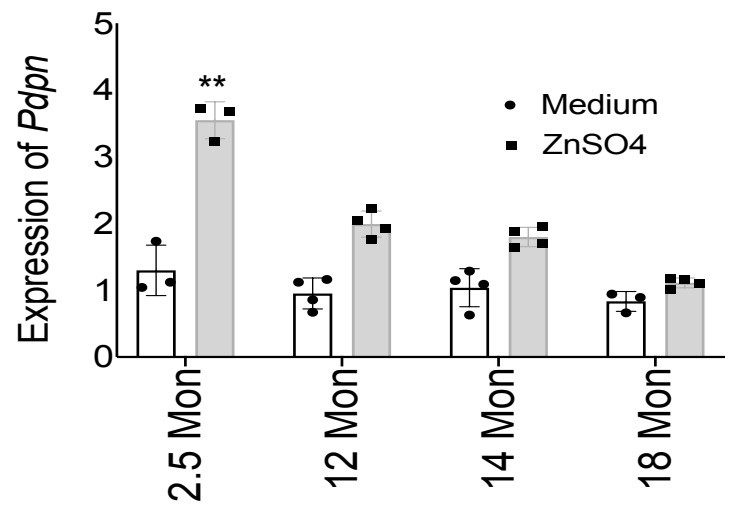

Figure 6. Aging blunted the response of AEC2s to exogenous zinc.

(A) CFE of AEC2s from uninjured wild type mice treated with exogenous $\mathrm{ZnSO} 4(100 \mathrm{mM})\left(\mathrm{n}=6\right.$, ${ }^{* *} \mathrm{p}<$ $0.01)$.

(B) Flow cytometry gated ZIP8+ AEC2s cultured with medium only or medium containing $100 \mathrm{mM}$ ZnSO4.

(C) Percent of ZIP8+ AEC2s ( $\left.n=6,{ }^{* * *} p<0.001\right)$.

(D) CFE of mouse AEC2s isolated from lungs of $2.5,12,14$, and 18-month-old mice $\left(n=3-4,{ }^{*} \mathrm{p}<\right.$ $\left.0.05,{ }^{* *} p<0.01,{ }^{* * *} p<0.001,{ }^{* * * *} p<0.0001\right)$.

(E-G) qPCR gene expression of Slc39a8 (D), Sftpc (E), and Pdpn (F) (n=3-4, *p<0.05, ** $p<0.01$, $\left.{ }^{* * *} p<0.001,{ }^{* * * *} p<0.0001\right)$. 
Fig 7
A

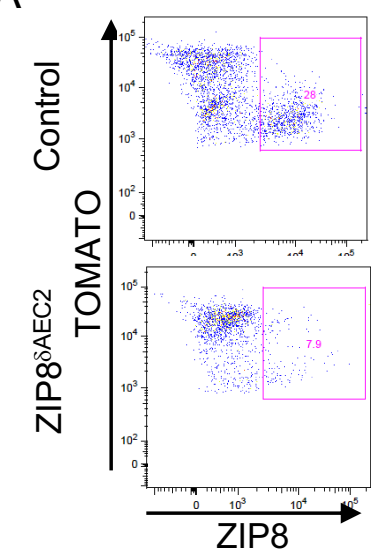

B

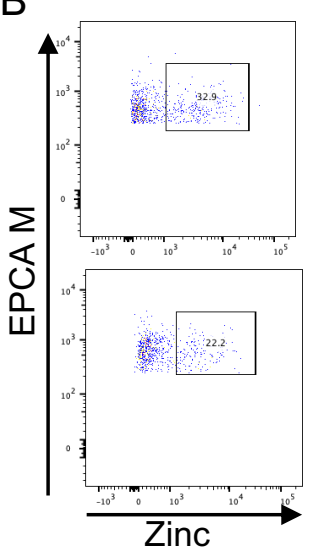

$\mathrm{C}^{\circ}$

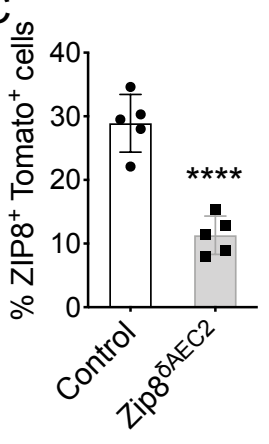

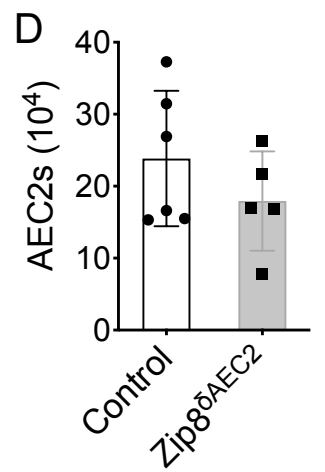

E

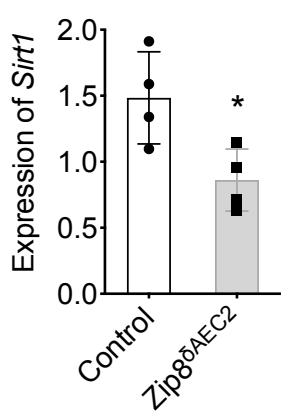

$\mathrm{F}$

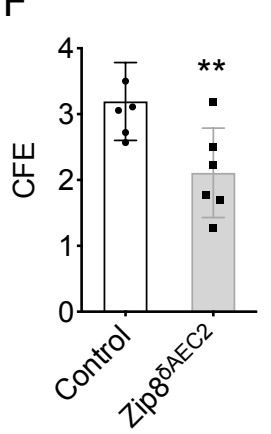

G

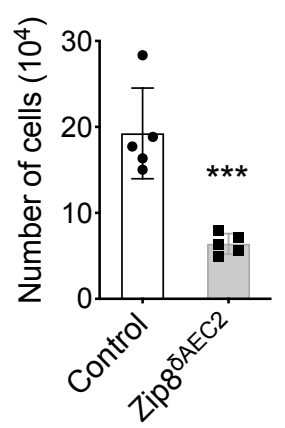

$\mathrm{H}$

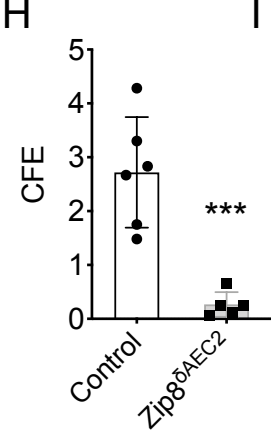

$\mathrm{L}$

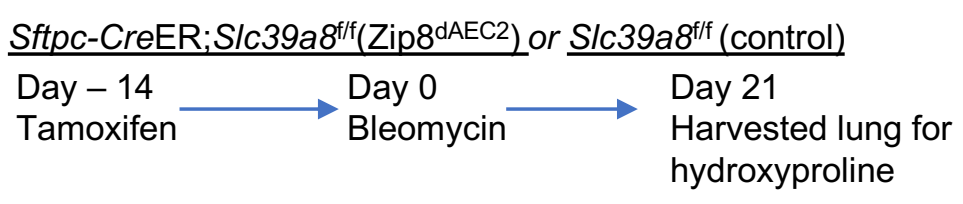

$\mathrm{N}$

Wild type mice

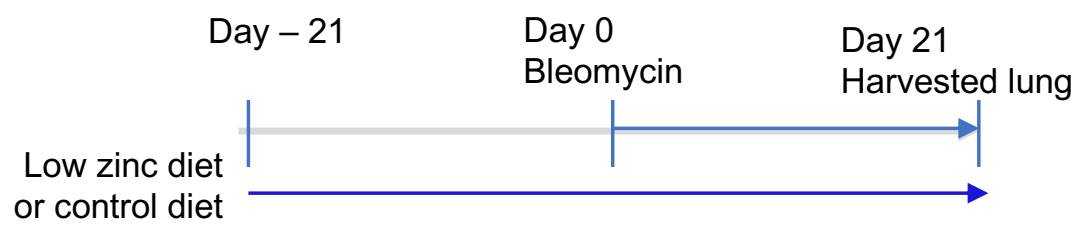

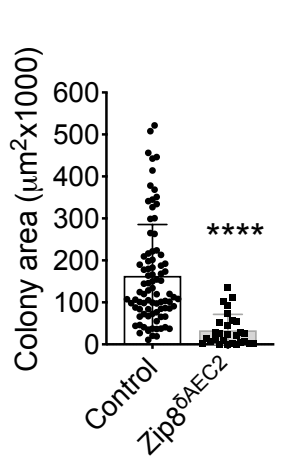

$J$

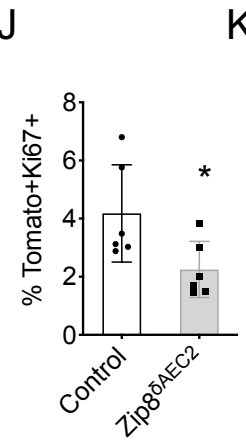

M

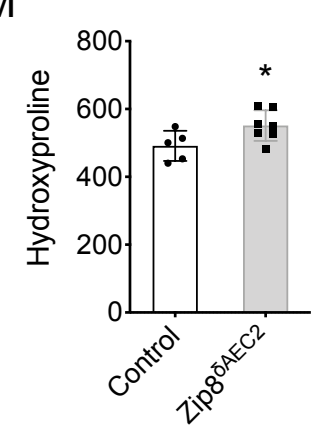

$\mathrm{O}$

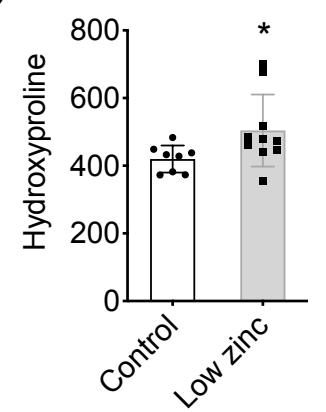

Figure 7. Targeted deletion of S/c39a8 decreased AEC2 renewal and worsened lung fibrosis. (A) ZIP8 expressing of AEC2s from Zip8 ${ }^{\triangle A E C 2}$ and control mice by flow cytometry. (B) Intracellular zinc in AEC2s from Zip8 ${ }^{\triangle A E C 2}$ (mean $25.03 \%, n=4$ ) and control mice (mean $36.10 \%, n=6$ ) by flow cytometry $(p<0.01)$. (C) ZIP8 expressing AEC2s $\left(\right.$ Tomato $^{+}$) from Zip8 ${ }^{\triangle A E C 2}$ and control mice by flow cytometry $\left(n=5\right.$ each, $\left.{ }^{* * * *} p<0.0001\right)$. (D) Number of AEC2s recovered from uninjured lungs of Zip8 ${ }^{\triangle A E C 2}$ and control mice $(n=6-8)$. (E) Sirt1 expression of AEC2s by qPCR $\left(n=4,{ }^{*} p<0.05\right)$. (F) CFE of AEC2s from uninjured mouse lungs $\left(n=6\right.$ each, $\left.{ }^{* *} p<0.01\right)$. (G) Number of AEC2 recovered per lung of Zip8 ${ }^{\triangle A E C 2}$ and control mice at day 4 after bleomycin $\left(n=5\right.$ each, $\left.{ }^{* *} p<0.01\right)$. $(H, I)$ CFE $(n=5-6)$ and colony size $(n=28-86)$ of AEC2s isolated from Zip8 $\triangle A E C 2$ and control mice at day 4 after bleomycin $\left(^{* * *} p<0.001,{ }^{* * * *} p<0.0001\right)$. (J) Ki67 expression of AEC2s derived from 3D cultured organoids of AEC2s from bleomycin injured Zip8 ${ }^{\triangle A E C 2}$ and control mice by flow cytometry $\left(n=6\right.$ each, $\left.{ }^{* *} p<0.05\right)$. (K) Pdpn expression of AEC2s derived from 3D cultured organoids of AEC2s from bleomycin injured Zip8 ${ }^{\triangle A E C 2}$ and control mice by qPCR ( $n$ $=5$ each, $\left.{ }^{* * *} p<0.0001\right)$. (L) Experiment layout of Zip8 ${ }^{\triangle A E C 2}$ and control mice treated with $1.25 \mathrm{U} / \mathrm{kg}$ bleomycin following tamoxifen injection. (M) Hydroxyproline levels of lungs from Zip8 ${ }^{\triangle A E C 2}$ and control mice harvested at day 21 after bleomycin $\left(n=5-7,{ }^{*} p<0.05\right)$. (N) Experiment layout of wild-type mice treated with $2.5 \mathrm{U} / \mathrm{kg}$ bleomycin following zinc deficient and control diet. (O) Hydroxyproline levels of lungs of mice fed with zinc deficient diet and control diet harvested at day 21 after bleomycin $\left(n=8-10,{ }^{*} p<0.05\right)$. 


\section{Figure S1}

A
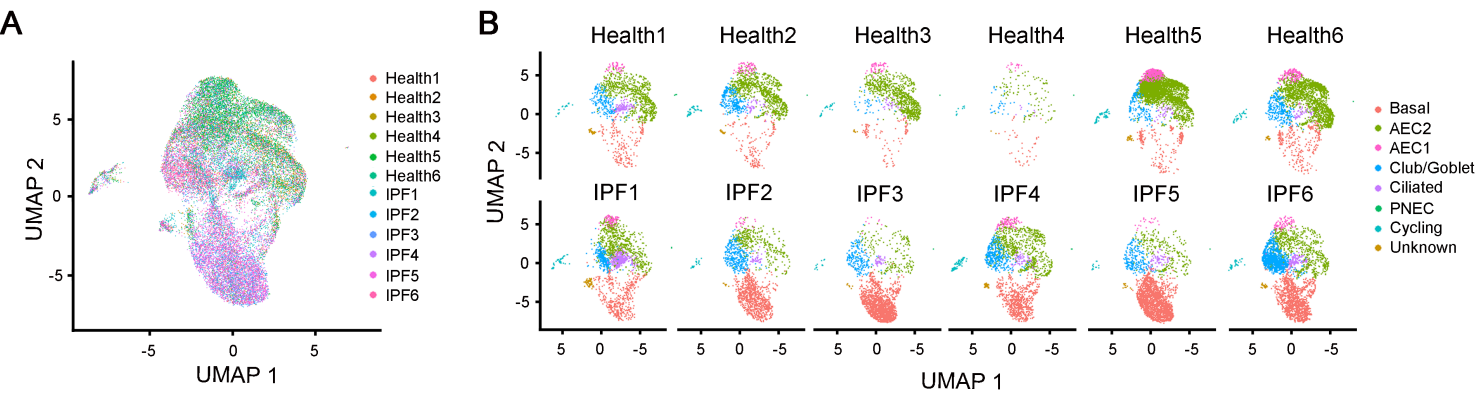

C

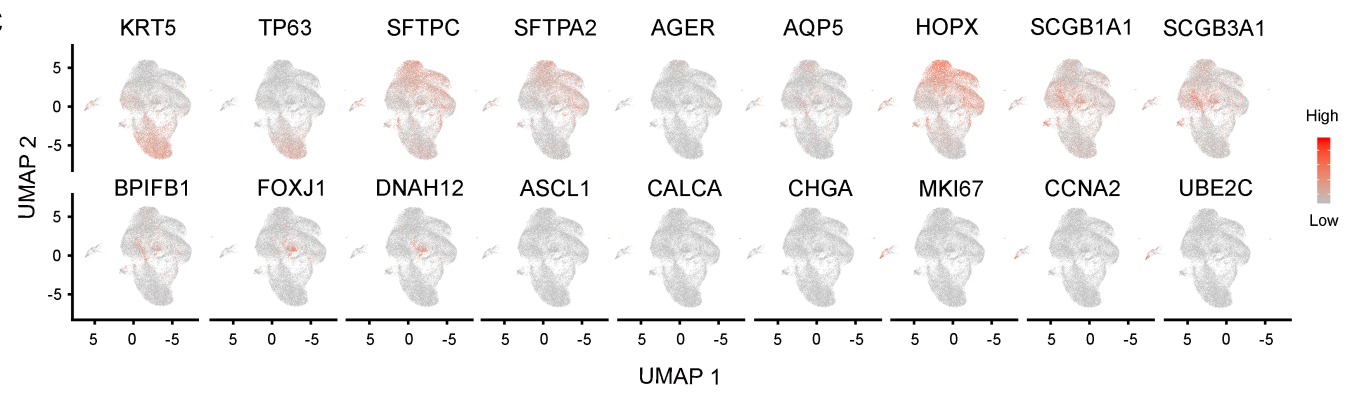

Figure S1. scRNA-seq analysis of lung epithelial cells.

(A) UMAP plots of lung epithelial cells from IPF and healthy donors ( $n=6$ each).

(B) Feature plots of lung epithelial clusters grouped by subject.

(C) Feature plots of lung epithelial clusters by classic cell type markers. 
$\underset{\mathrm{A}}{\text { Figure S2 }}$

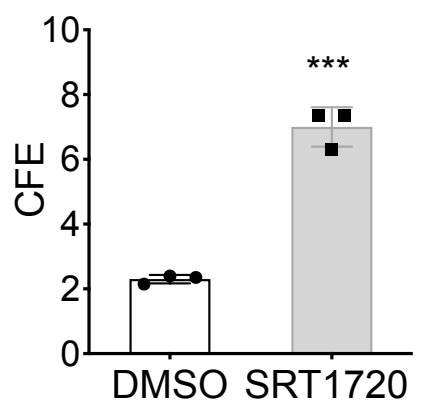

B

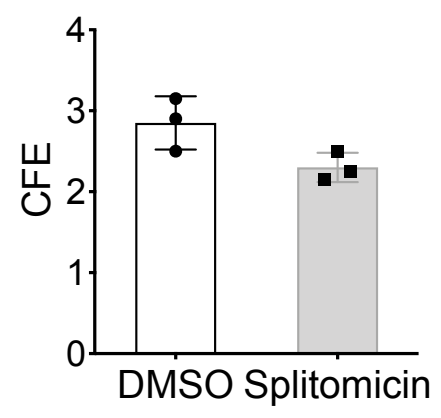

Figure S2. Effect of SIRT activator and inhibitor on mouse AEC2s.

$(A, B) C F E$ of $A E C 2$ from uninjured wild type mice treated with SRT $1720(A)$, splitomicin (B) with DMSO as control $\left(n=3\right.$ each. $\left.{ }^{* *} p<0.001\right)$. 


\section{Figure S3}

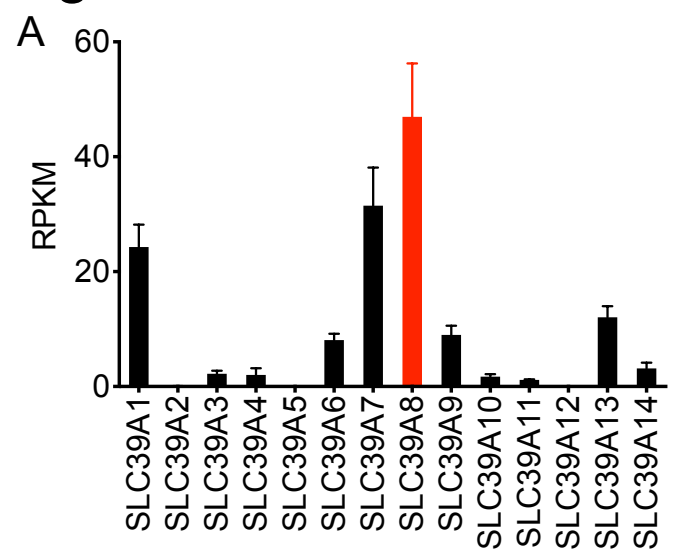

C

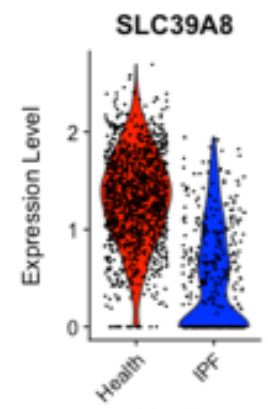

$\mathrm{D}$

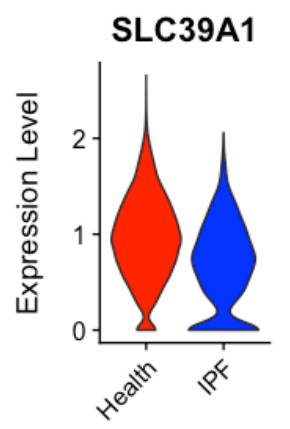

B

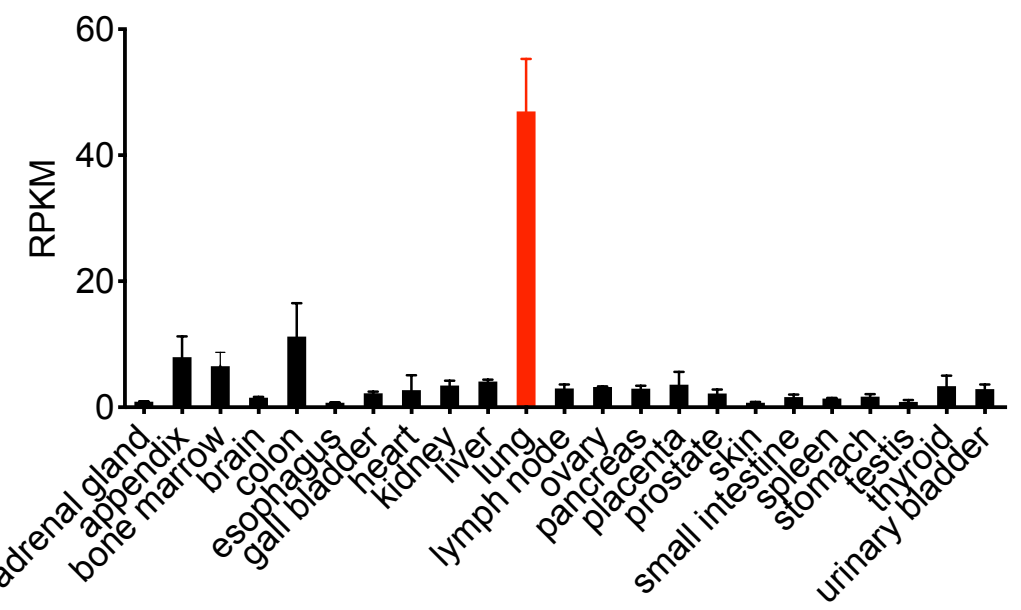

$\mathrm{E}$
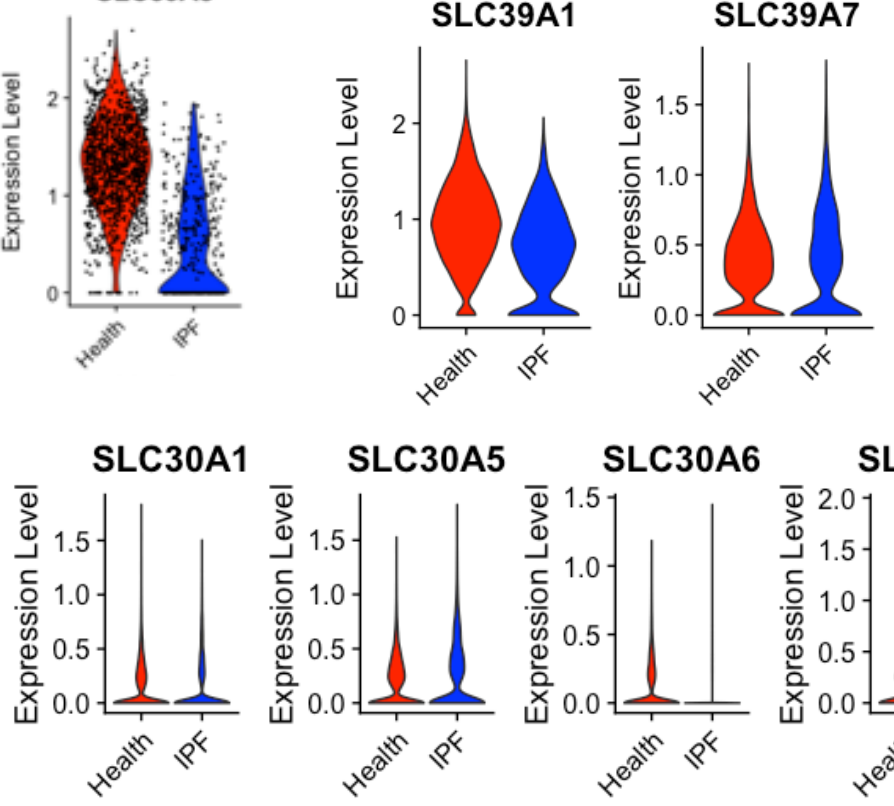

SLC39A7

$\mathrm{F}$
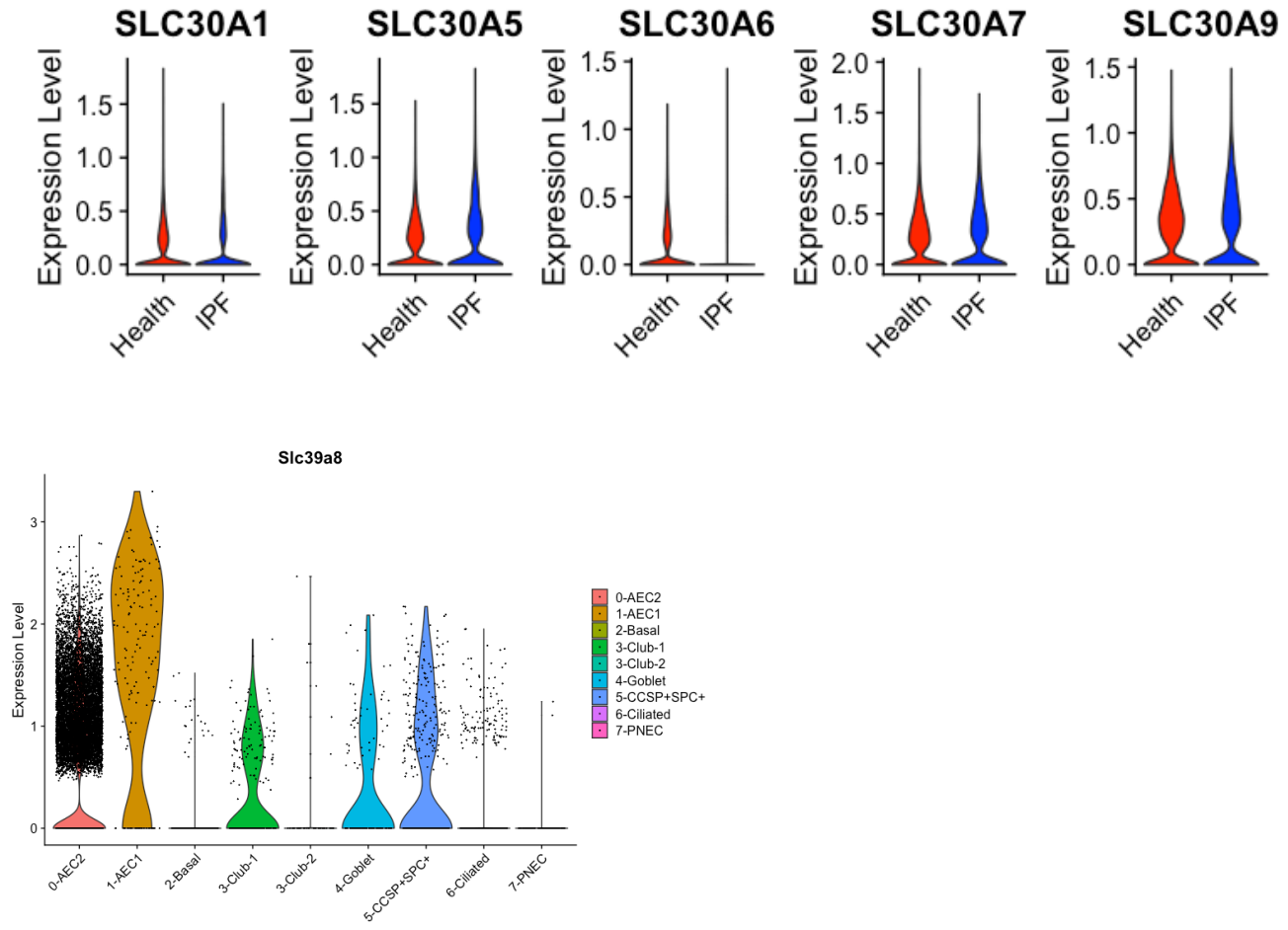

Figure S3. Specific expression of SLC39A8 in alveolar epithelial cells.

(A) Expression of SLC39A1-14 in lung tissue.

(B) Expression of SLC39A8 in human tissues and organs.

(C) Expression of SLC39A8 in AEC1s from healthy and IPF lungs.

(D) Expression of SLC39A1 and SLC39A7 in AEC2s from healthy and IPF lungs.

(E) Expression of ZNT family genes SLC30A1, SLC30A5, SLC30A6, SLC30A7, and SLC30A9

in AEC2s from healthy and IPF lungs.

(F) Expression of S/c39a8 in mouse lung epithelial cells. 


\section{Figure S4}

A

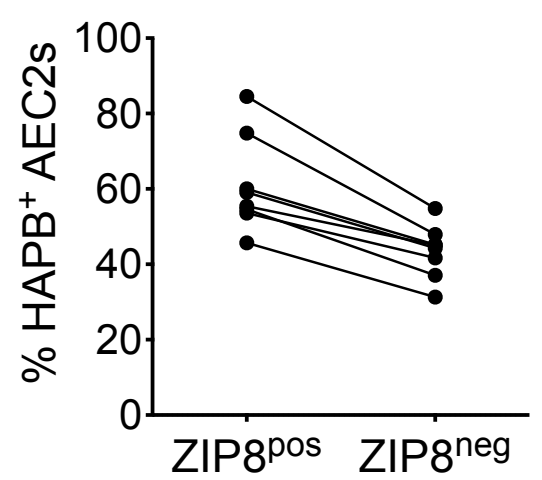

B

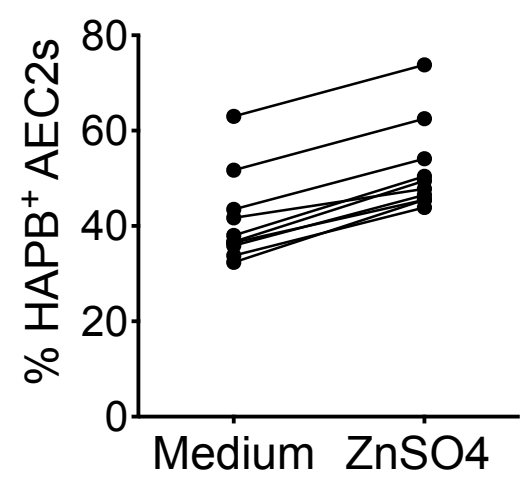

Figure S4. Correlation of ZIP8 and hyaluronan expression.

(A) Percent of $\mathrm{HABP}^{+}$of ZIP8neg and ZIP8pos ACE2s by flow cytometry.

(B) Percent of $\mathrm{HABP}^{+} \mathrm{AEC} 2 \mathrm{~s}$ with and without $100 \mathrm{~mm} \mathrm{ZnSO} 4$ treatment. 
bioRxiv preprint doi: https://doi.org/10.1101/2020.07.30.229567; this version posted July 30, 2020. The copyright holder for this preprint (which was not certified by peer review) is the author/funder. All rights reserved. No reuse allowed without permission.

\section{Figure S5}

A

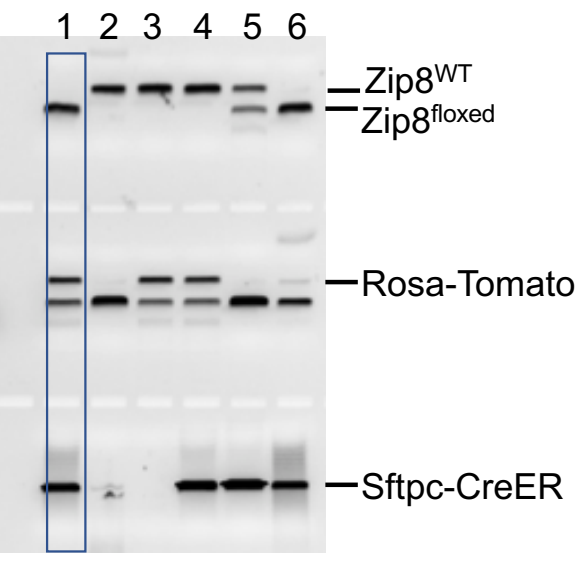

B

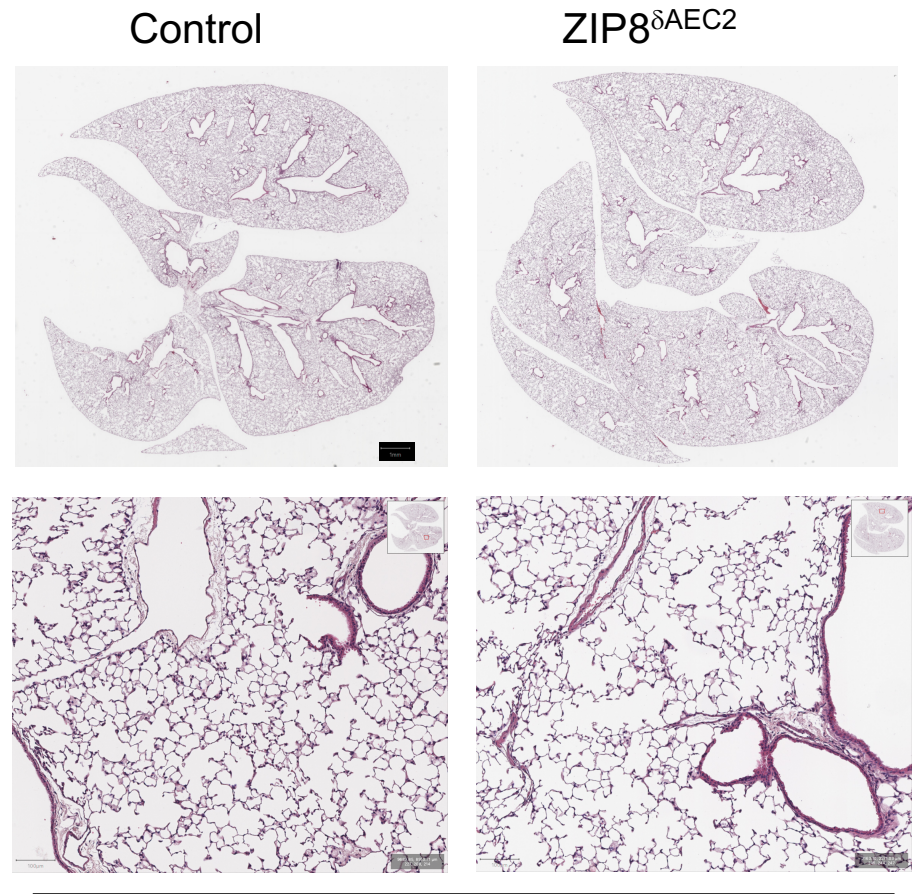

H\&E staining

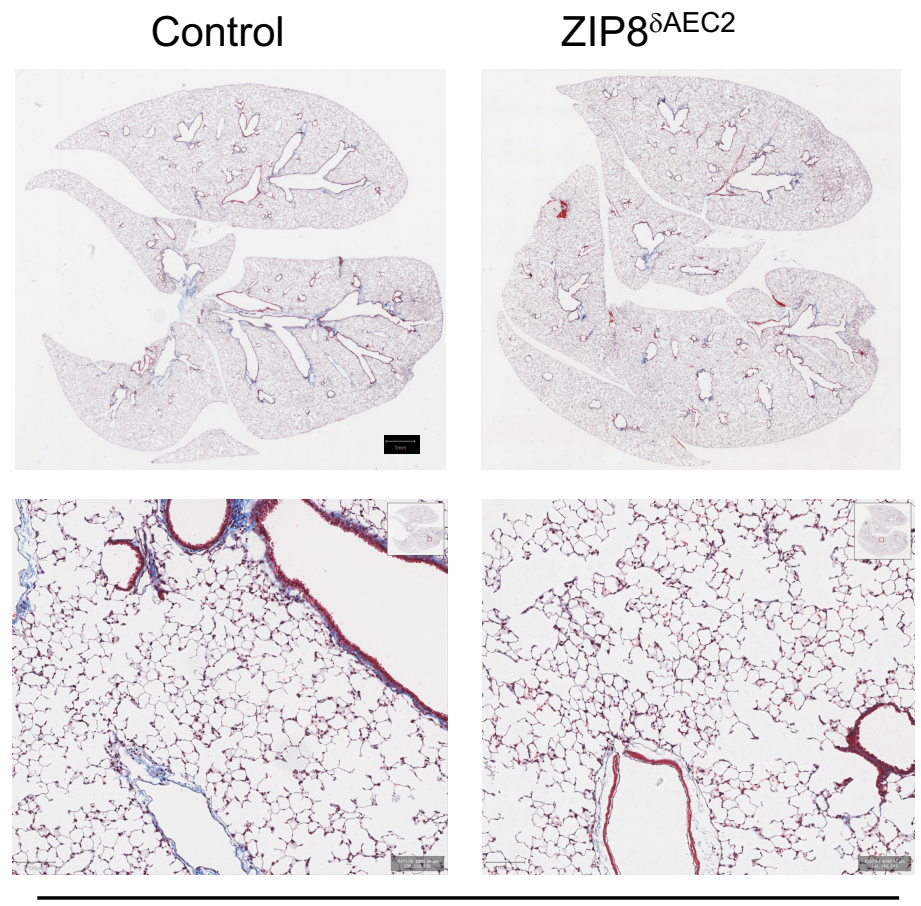

Trichrome staining

Figure S5. No obvious morphological defect in Zip8 deletion mice.

(A) A representative genotyping result of Sftpc-CreER ${ }^{+}$;RosaTomato+:Zip8flox/flox (Zip8 ${ }^{\triangle A E C 2}$ ) mice. $(B, C)$ Histological characterization (B, H\&E staining; $C$, trichrome) of uninjured lungs of Zip8 $\triangle A E C 2$ and control mice at $8-10$ weeks old. 